\title{
Stochastic Geometry Analysis of a Class of Cooperative Relaying Protocols
}

\author{
Sri Lakshmi Phani Balineni
}

Follow this and additional works at: https://researchrepository.wvu.edu/etd

\section{Recommended Citation}

Balineni, Sri Lakshmi Phani, "Stochastic Geometry Analysis of a Class of Cooperative Relaying Protocols" (2017). Graduate Theses, Dissertations, and Problem Reports. 5149.

https://researchrepository.wvu.edu/etd/5149

This Thesis is protected by copyright and/or related rights. It has been brought to you by the The Research Repository @ WVU with permission from the rights-holder(s). You are free to use this Thesis in any way that is permitted by the copyright and related rights legislation that applies to your use. For other uses you must obtain permission from the rights-holder(s) directly, unless additional rights are indicated by a Creative Commons license in the record and/ or on the work itself. This Thesis has been accepted for inclusion in WVU Graduate Theses, Dissertations, and Problem Reports collection by an authorized administrator of The Research Repository @ WVU. For more information, please contact researchrepository@mail.wvu.edu. 


\title{
Stochastic Geometry Analysis of a Class of Cooperative Relaying Protocols
}

\author{
Sri Lakshmi Phani Balineni \\ Thesis submitted to the \\ College of Engineering and Mineral Resources \\ at West Virginia University \\ in partial fulfillment of the requirements \\ for the degree of \\ Master of Science \\ in \\ Electrical Engineering \\ Brian D. Woerner, Ph.D. \\ Daryl S. Reynolds, Ph.D. \\ Matthew C. Valenti, Ph.D., Chair \\ Lane Department of Computer Science and Electrical Engineering \\ Morgantown, West Virginia \\ 2017
}

Keywords: Stochastic geometry, Hybrid-ARQ, Cooperative relaying, Outage probability, Throughput, Spatial averaging

Copyright 2017 Sri Lakshmi Phani Balineni 


\section{Abstract \\ Stochastic Geometry Analysis of a Class of Cooperative Relaying Protocols \\ Sri Lakshmi Phani Balineni}

This thesis examines wireless relay networks that use hybrid-ARQ protocols. Relays networks efficiently combat fading and exploit the spatial diversity present in the channel. Hybrid-ARQ involves re-transmitting the signal if it is not decoded correctly. In conventional HARQ, the re-transmission comes from the source, but in cooperative HARQ the re-transmission could come from a relay that has successfully decoded the message, thus attaining transmit diversity.

A Markov chain model is conceived and used to compute the effective throughput and outage probability in the presence of Rayleigh fading. The analytical results are validated with simulations. The spatial configuration of the network plays an important role in the performance of the network. The behavior of the protocols for fixed network topologies and random topologies is examined. The impact of parameters such as path loss exponent, number of relays, and Signal to Noise Ratio are determined.

Spatial averaging is helpful in capturing the spatial variations present in the system. When network topology is random, the analysis proceeds by first assuming the number of relays is fixed, in which case they are drawn from a Binomial Point Process (BPP). For each network realization, the outage probability, throughput and effective throughput are found, and the spatial average of these quantities are found by averaging over a large number of network realizations. Moreover, the maximum throughput is found for each network realization, leading to a characterization of the distribution of throughputs achievable in a random network. Finally, networks with a random number of relays are considered, including the important case that the number of relays in a given area is Poisson distributed, in which case they are drawn from a Poisson Point Process (PPP). 


\section{Acknowledgements}

First, I want to thank my committee chair and advisor, Dr. Matthew C. Valenti, for guiding me in my research and providing me an opportunity to work with him. He has been very patient and supportive. Our weekly meetings enhanced my coding skills, thought process and approach towards the research problem. I am indebted for all his help.

I am thankful to Dr. Daryl Reynolds and Dr. Brian Woerner for accepting to serve on the committee immediately when I approached them, despite their busy schedule. Dr. Reynolds has been an excellent teacher, his lectures strengthened my concepts in communication systems.

Among other faculty, Dr. Noore needs a special mention, he recognized my skills and offered me GTA position. He always motivated me to pursue thesis option. I would also like to thank Terry Ferret, for helping me to get started with the WCRL cluster and clarifying my doubts on WCRL cluster.

I am also thankful to my friends here at WVU, who encouraged and supported me. Last but not the least, I remain indebted to my parents Rama Devi and Raghunadha Rao, without their love, support and encouragement nothing would have been possible. 


\section{Contents}

$\begin{array}{ll}\text { Acknowledgements } & \text { iii }\end{array}$

List of Figures

Notation $\quad$ vii

1 Introduction $\quad 1$

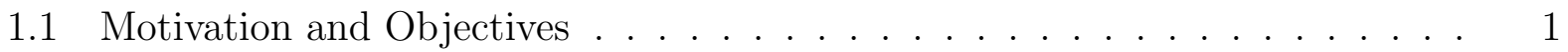

1.2 Thesis Outline . . . . . . . . . . . . . . . . . . . . 2

2 Literature Review $\quad 4$

2.1 Relay Networks . . . . . . . . . . . . . . . . . . . . . . . 4

2.2 Outage Behavior in Relay Networks . . . . . . . . . . . . . . . . 6

2.3 Spatially Averaged Outage Probability . . . . . . . . . . . . . . . . 6

2.4 Automatic Repeat reQuest . . . . . . . . . . . . . . . . . . . 7

2.5 Hybrid Automatic Repeat reQuest . . . . . . . . . . . . . . . . 8

2.6 Cooperative Hybrid ARQ . . . . . . . . . . . . . . . . . . 9

3 System Model and Protocol 10

3.1 System Model . . . . . . . . . . . . . . . . . . . . . . . . . . 10

3.2 Protocol Description . . . . . . . . . . . . . . . . . . . . . . 12

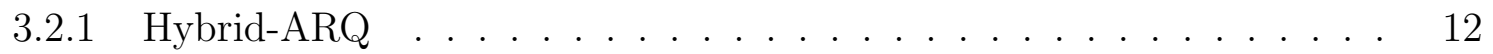

3.2 .2 Cooperative Hybrid-ARQ . . . . . . . . . . . . . . . . . . . 12

3.3 Channel parameters . . . . . . . . . . . . . . . . . . . . . . 14

3.3.1 Path Loss Exponent . . . . . . . . . . . . . . . . . . 14

3.3.2 Rayleigh Fading . . . . . . . . . . . . . . . . . . . . . 14

3.3.3 Outage Threshold and Probability . . . . . . . . . . . . 15

3.4 Simulation . . . . . . . . . . . . . . . . . 16

4 Throughput Analysis of Fixed Networks 18

4.1 Analysis of Line Networks Using Markov Chains . . . . . . . . . . . . . . . 19

4.1 .1 No Relays . . . . . . . . . . . . . . . . . . . . . . . . . . . . 19

4.1 .2 One Relay . . . . . . . . . . . . . . . . . . . . 20

4.1 .3 Multiple Relays . . . . . . . . . . . . . . . . . . . . 24

4.2 Outage Probability and Effective Throughput . . . . . . . . . . . 26 
4.2.1 Outage Probability . . . . . . . . . . . . . . . . 26

4.2.2 Throughput and Effective Throughput . . . . . . . . . . . 26

4.2 .3 Numerical results . . . . . . . . . . . . . . . . . . 27

5 Spatial Averaging and Random Networks $\quad 32$

5.1 Random Networks . . . . . . . . . . . . . . . . . . . . . . . . 32

5.2 Effect of Network Topology on System Performance . . . . . . . . . . . . 33

5.3 Spatial Average . . . . . . . . . . . . . . . . . . 33

5.3.1 Spatial Averaging when the Network is BPP defined over Region . . . 35

5.3.2 Poisson Point Process . . . . . . . . . . . . . . . . . . . 35

5.3.3 Numerical Results . . . . . . . . . . . . . . . . 36

6 Conclusions and Future Work 43

6.1 Summary of Contributions . . . . . . . . . . . . . . . . . . . 43

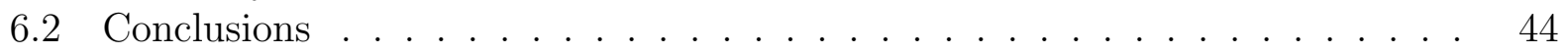

6.3 Future Work . . . . . . . . . . . . . . . . . . 44

$\begin{array}{ll}\text { Bibliography } & 45\end{array}$ 


\section{List of Figures}

2.1 Classic Relay Network. . . . . . . . . . . . . . . . . . . . . 5

3.1 A simple M-Relay Network with two hops. . . . . . . . . . . . . . . 11

3.2 Flowchart for simulation. . . . . . . . . . . . . . . . . . . 17

4.1 Simple line network . . . . . . . . . . . . . . . . . . . . . . . 18

4.2 Markov chain for no relays case. . . . . . . . . . . . . . . . . . . . . . 19

4.3 Trellis diagram for no relays case . . . . . . . . . . . . . . . . . . . . . . . . . . . . . . . . . . . . . .

4.4 Markov Chain for one relay. . . . . . . . . . . . . . . . . . . . 21

4.5 Trellis diagram for one relay. . . . . . . . . . . . . . . . . . . . . . . 22

4.6 Markov chain for multiple relays case. . . . . . . . . . . . . . . . . . . . 25

4.7 Trellis diagram for multiple relays. . . . . . . . . . . . . . . . . . . . 26

$4.8 P_{\text {out }}$ for the change in $\mathrm{M}$ when $\alpha=3.5$ and $\Gamma=30 \mathrm{~dB}$. . . . . . . . . . 29

4.9 Effective throughput for change in $\mathrm{M}$ when $\alpha=3.5$ and $\Gamma=30 \mathrm{~dB}$. . . . . 29

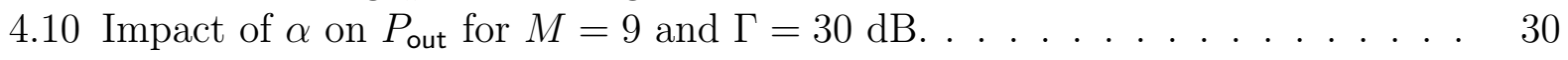

4.11 Impact of $\alpha$ on $\eta_{\text {eff }}$ for $M=9$ and $\Gamma=30 \mathrm{~dB}$. . . . . . . . . . . . . . . . 30

4.12 Impact of $\Gamma$ on $P_{\text {out }}$ for $M=9$ and $\alpha=3.5 \ldots \ldots$. . . . . . . . . . 31

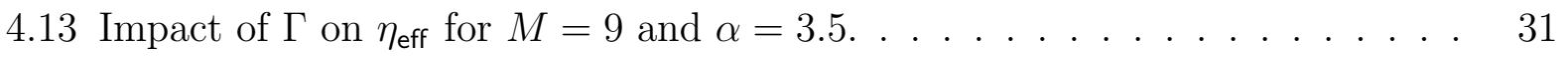

5.1 Random network model. . . . . . . . . . . . . . . . . . . . 33

5.2 Effect of random placement of relays on $P_{\text {out }} \ldots \ldots . . \ldots 34$

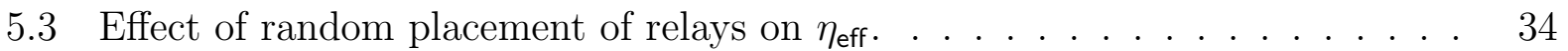

5.4 Spatially averaged outage probability for $\Gamma=30 \mathrm{~dB}$ and $\alpha=3.5$. . . . . . . 38

5.5 Spatially averaged effective throughput for $\Gamma=30 \mathrm{~dB}$ and $\alpha=3.5$. . . . . 38

5.6 Spatially averaged outage probability for $\alpha=3.5$ and $M=10$. . . . . . . . 39

5.7 Spatially averaged effective throughput for $\alpha=3.5$ and $M=10$. . . . . . . 39

5.8 Spatially averaged outage probability for $M=10$ and $\Gamma=30 \mathrm{db}$. . . . . . 40

5.9 Spatially averaged effective throughput for $M=10$ and $\Gamma=30 \mathrm{db}$. . . . . 40

5.10 Spatially averaged outage probability for PPP. . . . . . . . . . . . . 41

5.11 Spatially averaged $\eta_{\text {eff }}$ for PPP. . . . . . . . . . . . . . . . . . 41

5.12 Cumulative distribution of maximum throughputs for BPP case. . . . . . . 42

5.13 Cumulative distribution of maximum throughputs for PPP case. . . . . . . 42 


\section{Notation}

We use the following notation and symbols throughout this thesis.

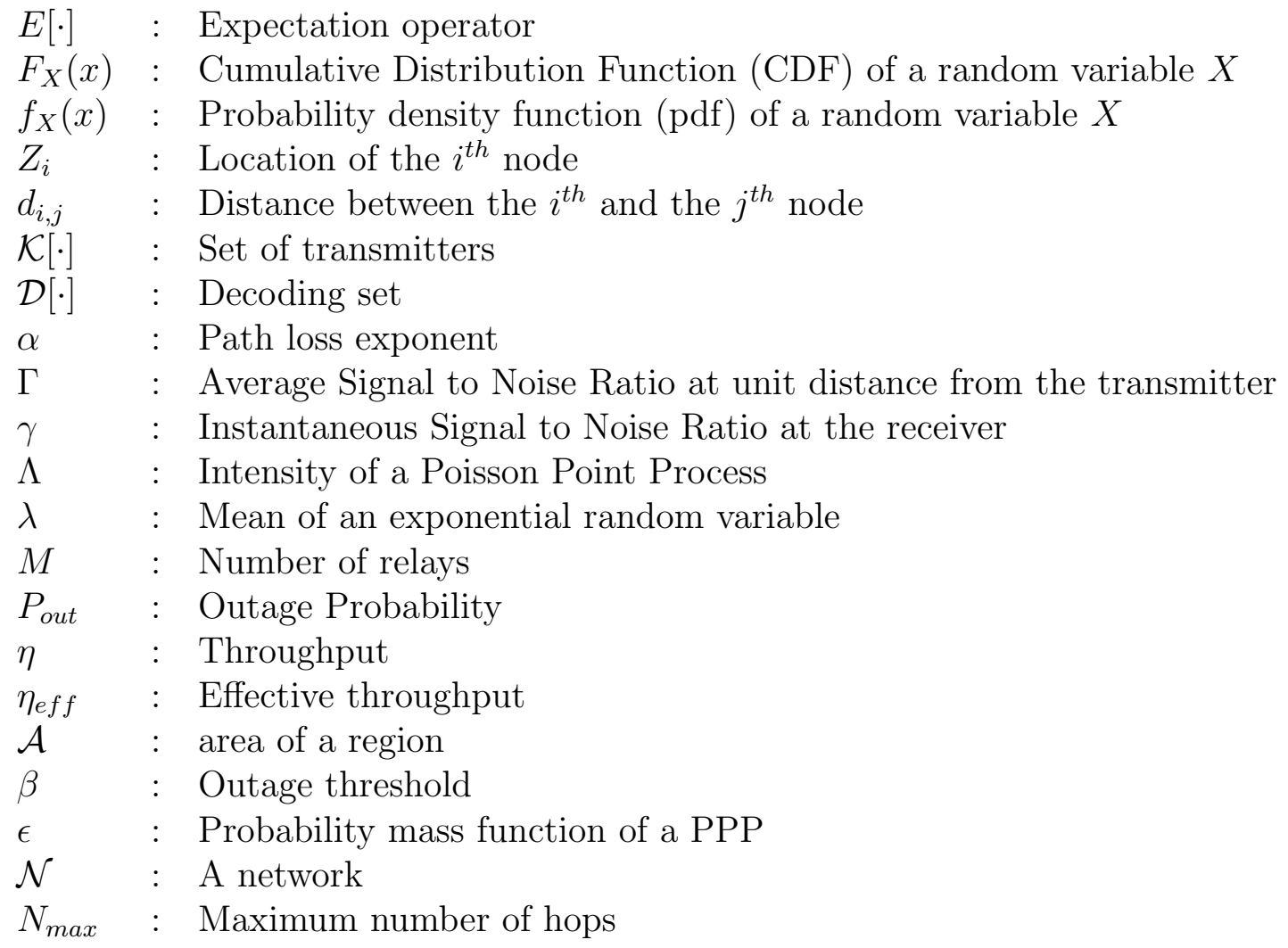




\title{
List of Abbreviations
}

\author{
ARQ : Automatic Repeat reQuest \\ BPP : Binomial Point Process \\ CDF : Cumulative Distribution Function \\ FEC : Forward Error Correction \\ GeRaF: Geographic Random Forwarding \\ HARQ : Hybrid Automatic Repeat reQuest \\ HARBINGER: Hybrid-ARq-Based Intracluster GEographic Relaying \\ pdf : Probability Distribution Function \\ PPP : Poisson Point Process \\ SNR : Signal to Noise Ratio
}




\section{Chapter 1}

\section{Introduction}

This chapter gives an overview of cooperative networks, relaying, and ARQ protocols. The main objectives and the outline of the thesis are explained in this chapter.

\subsection{Motivation and Objectives}

Wireless technology has evolved at a rapid pace in the past decades. This advancement has not only lead to the increase in the number of users but also in the improvement of the quality of communication. Research focus has shifted to improving the system performance in degraded environments with severe fading and using bandwidth efficiently. The resources present in the channel should be utilized in such a way that they maximize the data transmission rate with minimum complexity and cost efficiency.

Cooperative communications involves the use of one or more relays assisting the other nodes in transmitting the data to the destination. Cooperative communications essentially create a virtual antenna array to assist in signal transmission. The spatial diversity of the channel is exploited. There are various relaying strategies, of which the decode-and-forward scheme is analyzed and implemented in this thesis [1] [2]. Cooperative communication protocols are modelled as a relaychannel, which efficiently combat signal fading [3] [4].

Automatic Repeat reQuest (ARQ) protocols are known for providing reliable transmission. In an ARQ protocol, if the transmitted signal is not decoded correctly then a retransmission is made. Several cooperative ARQ protocols have been proposed and their performance is analyzed [5] [6] [7]. Hybrid ARQ could be considered as an advancement of basic ARQ protocol, as it incorporates both forward error correction and detection. If the 
packet is successfully decoded at the receiver, in other words when the transmission is free from errors, then the receiver requests for a new packet transmission. On the other hand, if the receiver is unable to correctly decode the packet, then a re-transmission is requested. Hybrid ARQ is efficient in achieving higher throughput because of the forward error correction and lesser number of re-transmissions. In conventional HARQ, the re-transmission comes from the source, but in cooperative HARQ the re-transmission could come from a relay that has successfully decoded the message, thus attaining the transmit diversity.

Hence, the major objective of this thesis is to use Hybrid-ARQ protocol in conjunction with relay networks and to optimize the system performance. In order to achieve this the protocol is tested for two different network topologies and under the influence of channel fading parameters.

\subsection{Thesis Outline}

This thesis deals with hybrid-ARQ protocol and relay networks. Chapter 2 begins with the description of the system model and HARQ protocol. First, the HARQ protocol is explained, then the difference between cooperative HARQ and conventional HARQ is stated. The protocol described in the thesis is similar to HARBINGER and GeRaF protocols in [8] [9]. The difference among GeRaF, HARBINGER, and the protocol used in thesis is explained. Chapter 2 also discusses the simulation of the protocol.

Chapter 3 discusses the implementation of the protocol for line networks. In particular the Markov chains and trellis diagrams that are used to compute the outage probability and effective throughput are discussed. The outage probability and effective throughput are analyzed under the influence of several key parameters parameters such as the path loss exponent, number of relays and SNR.

Chapter 4 is specific to random networks. The random network model chosen is described. The influence of the topology on the network performance is discussed, leading to the concept of spatial averaging. A direct method to compute spatial average is adopted from [10]. The spatially averaged outage probability and effective throughput are determined for two cases. In the first case, the number of relays is fixed, and the relays are drawn from a Binomial Point Process (BPP). Then networks with random number of relays are considered; given that the number of relays in a given area are Poisson distributed and they are drawn from a Poisson Point Process (PPP). 
The final chapter summarizes the important results obtained and the conclusions. The possible ways in which this work can be extended is presented in a section on future work. 


\section{Chapter 2}

\section{Literature Review}

This chapter presents a review of research efforts in the area of cooperative communications and relay networking. The research works involving ARQ and hybrid-ARQ protocols are discussed in relation to the work and results presented in this thesis.

\subsection{Relay Networks}

A wireless network basically consists of atleast a transmitter and receiver. The transmitter is also termed as a source because it has the message and the receiver is also called a destination. A direct link from the source to destination is not always feasible, especially when the transmitter and receiver are separated by a large distance. In this case, the source needs to make high power transmissions to facilitate a direct link, which leads to inefficient utilization of power resources and faster battery drain in case of mobiles. It is shown in [11] that an order-of-magnitude reduction is possible in exploiting the spatial diversity in ad-hoc networks. To mitigate shortcomings of direct links, relay channels are introduced. In a relayed transmission, the message propagation takes place with the help of relays that are located in the region between transmitter and receiver. The concept of the relay channel model dates back to the early 1970's. The original ideas on relay channels can be traced back to the work of Cover and El Gamal [12], their analysis is mainly on the capacity of the relay channel and various theorems are formulated in this work. Later, Laneman, Tse and Wornell [1] described three different relay behaviors that can be possible in the discrete memory less and additive white Gaussian noise relay channels.

A good analysis of wireless network architectures that utilize relays is presented in [13]. It 


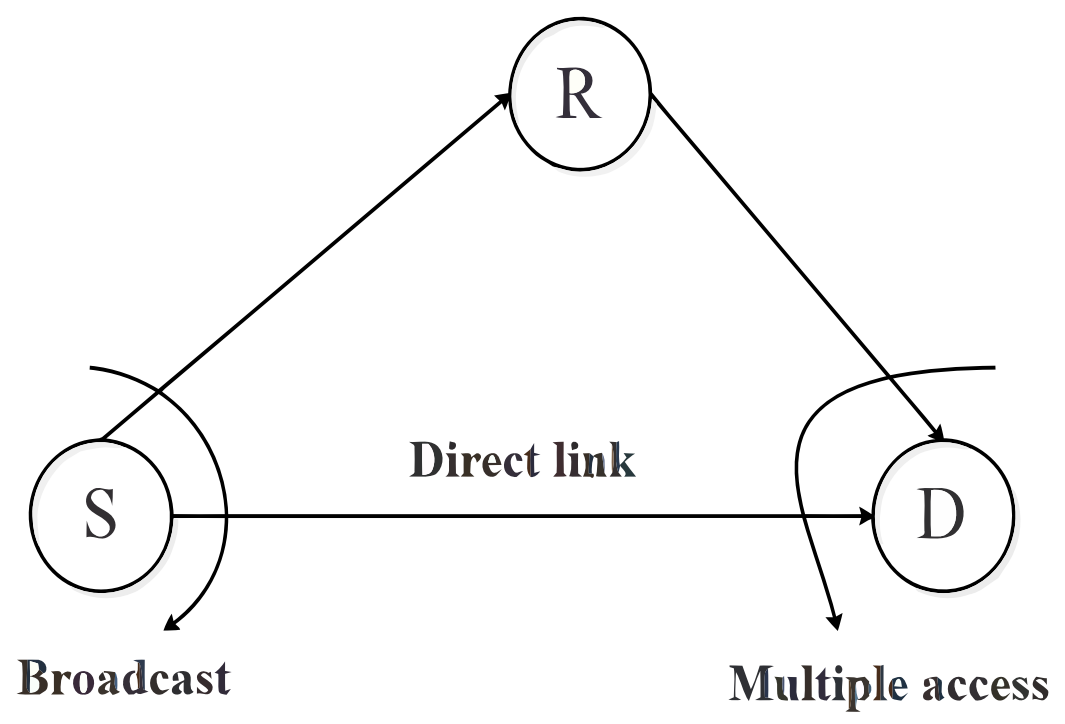

Figure 2.1: Classic Relay Network.

describes the prevalent wireless network architectures and specifies the new architectures of relay channels that can be incorporated into existing architectures. The simplest description of a relay channel consists of three terminals: a source, a destination and a relay. It is also called classic relay channel [13], in a classic relay channel shown in fig.2.1 the source broadcasts its transmissions to relays and destination simultaneously. The transmissions from the source are considered to be broadcast in nature and the destination perceives the relay channel to be a multiple access channel since it receives transmission from both the source and relay. This thesis work assumes the usage of the classic relay channel with multiple relays.

Three types of relaying, namely: fixed, selection and incremental, are described by Laneman [1]. In fixed relaying, the relay that receives the message signal will either amplify it and retransmit or fully decodes, re-encodes and re-transmits it. Selective relaying protocols determine the best path to the destination by following a particular strategy for making the selection. Fixed and selective relaying are not efficient for high rates because relays always repeat even if the destination correctly received the message from the source over the direct path. Incremental relaying uses feedback from the destination regarding the success or failure of the direct transmission, and this feedback in turn improves the efficiency. We consider incremental relaying strategy, since the ACK/NACK of the ARQ protocol provides the feedback. Hence no relaying is necessary if the destination sends an ACK. It is assumed that 
the relays are capable of receiving, decoding, transmitting and re-encoding the signal. The protocol that involves selecting a best relay from the decoding set designed by Bletsas[14] also called opportunistic relaying. In opportunistic relaying, the best suitable relay with transmitter-relay-receiver link is selected based on the instantaneous channel measurements.

\subsection{Outage Behavior in Relay Networks}

Outage occurs when the SNR is below some minimum threshold required for the decoder to reliably decode the message. Papers [14][15][16] analyze the outage performance of a cooperative network under decode and forward relaying scheme and different constraints. [14] presents opportunistic relaying with decode and forward scheme under aggregate power constraint. It considers two relay selection strategies named as reactive and proactive relay selection based on whether the relay selection is performed before or after source transmission. [15] uses Laplace transform approach to find a closed form expression for outage probability by assuming independent and non-identically distributed channels. It analyzes the variation of outage probability when the Signal to Noise ratio increases and when the number of relays increases. The authors also prove that while using time division channel allocation scheme, the outage increases as the number of relays increases. [16] derives a closed form expression for outage probability for adaptive decode-and-forward relay network. In adaptive relay selection the number of relays in the decoding set is minimized while satisfying the outage requirement. A decoding set comprises of the set of nodes that have successfully decoded the message.

\subsection{Spatially Averaged Outage Probability}

Distance is a critical parameter in wireless networks since signal power and interference are dependent on the signal transmission distance. Hence, the spatial configuration of the network plays an important role in determining the performance of any network, including the relay networks. A system cannot be designed for a specific spatial configuration because different configurations are possible, therefore, a statistical spatial model must be considered for the node locations in a wireless network [17]. In large wireless systems with relays, classical methods of communication theory are inadequate [18]. Stochastic geometry deals with the random spatial patterns, by assuming that the network is modelled as a spatial 
point process. Point process theory is regarded as the sub field of stochastic geometry [18]. A point process can be viewed as a random collection of points in space.

Outage probability is only capable of characterizing the performance of a system with nodes in a particular location, but spatially averaged outage probability is helpful in capturing the spatial variations of the system due to the random movement and activity of the nodes. Five major techniques in the literature can be used to determine the spatially averaged outage probability. A simple overview of these five techniques is described by Valenti et al in [10] [19]. In this thesis, we use the direct approach to compute the spatially averaged outage probability as described in [10], with an important distinction being that [10] is concerned with randomly located interferers while we are concerned with randomly located relays. First,the relays are assumed to be in fixed positions and the outage probability is determined conditioning on the network topology, assuming Rayleigh fading. Then, the relays are allowed to assume random locations (according to a BPP) and the outage probability found out earlier is averaged with respect to the random positions of the relays. Finally, the BPP outage probability, which is calculated in step 1, is marginalized to the random location of relays drawn from a PPP, by allowing the number of relays to be random (i.e., Poisson in the case of PPP). The advantage of using the direct approach is that it can be extended to any arbitrary shaped network.

\subsection{Automatic Repeat reQuest}

In ARQ scheme if the transmitted message is received with errors, then the receiver automatically discards the message and asks for a retransmission. Retransmissions occur until the message is received correctly or for a preset number of times. The presence of errors is detected with the help of an error detecting code. Primarily, there are three types of ARQ protocols: stop-and-wait ARQ, go-back-N ARQ and selective-repeat ARQ [20]. A survey of three basic ARQ schemes and their performance; i.e., reliability and throughput analysis is presented in [20]. Apart from ARQ this paper also explains discusses the FEC error control systems but as they are less reliable, generally ARQ systems are preferred over FEC systems. The one major drawback of the basic ARQ schemes is that with the increase in the error rate, throughput decreases swiftly. One way of efficient error control is with the use of hybrid ARQ, which is discussed in the next section.

Numerous cooperative ARQ protocols have been proposed and their performance is an- 
alyzed in terms of throughput and bit error rate [5]. [6] proposes three cooperative ARQ protocols and their performance analysis in terms of packet error rate. However [6] analyzes the case of only one re-transmission. [7] investigates on the throughput performance for cellular networks with ARQ protocol by using Markov chains, this work considers the case of only one relay.

\subsection{Hybrid Automatic Repeat reQuest}

Hybrid ARQ combines the benefits of forward-error-correction coding with ARQ. Hybrid ARQ was first introduced in 1960's by Wozencraft and Horstein [21] [22]. With Hybrid-ARQ the message is encoded with both an error detecting code and an error correcting code. The receiver first tries to correct errors using the error correcting code, but if it fails, the error detecting code will let the receiver know there are residual errors. The receiver will then request a re-transmission, as in standard ARQ. The key difference is that the re-transmission rate can be significantly reduced by using an appropriate error correction code.

Hybrid ARQ gives better performance because error control coding and ARQ schemes are combined. FEC reduces the number of retransmissions by correcting the recurring error patterns and thereby improves the system throughput. However, if a less frequent error pattern occurs, then the receiver asks for retransmission. This improves the reliability of the system [20]. Hybrid ARQ schemes are of two types: type-I HARQ and type-II HARQ. In type-I, if the receiver cannot successfully decode the message, it discards it and asks for a re-transmission. The transmitter sends the code word and the receiver will attempt to decode using only the re-transmitted code word. This thesis implements protocol that uses type-I HARQ.

Type-II HARQ was introduced by Lin and Yu [23], later modified in [24]. In type-II, information is kept at the receiver when it fails to decode the message. The re-transmitted information is combined with the previously received information. The re-transmission can be either the same code word, in which case the old and new transmissions are diversity combined. Alternatively, the re-transmission could be different parity bits from a low rate error control code. The newly received code bits are combined with the previous code bits to create a virtual code of lower rate. 


\subsection{Cooperative Hybrid ARQ}

In conventional HARQ re-transmission comes from the source, but in cooperative HARQ re-transmission could come from any cooperating node in the network. For instance, if the source transmission is overheard by the relay that lies in between source and destination, then that relay could re-transmit the message rather than the source. The throughput of truncated cooperative hybrid-ARQ systems is analyzed with the help of Markov chains in [25]. There are many works that describe a variety of HARQ protocols and analyze their throughput performance, but a closed form expression for throughput is given in [25]. This thesis investigates the throughput and outage performance of the network, where the outage analysis of the network is done with the help of Markov chains.

A cooperative communication channel using ARQ is proposed by El. Gamal et al [26]. The protocol proposed in the paper achieves full diversity and full multiplexing gains. A generalization to the HARQ protocol in which the retransmitted packets originate from any relay that has overheard the message signal and can successfully decode the message is considered by Valenti and Zhao in [8]. The protocol described in [8] is termed as hybrid-ARQ-based intracluster geographic relaying (HARBINGER). A good contrast between HARBINGER, instantaneous relaying and multihop protocols is presented in this paper. A modified version of HARBINGER protocol is presented in [27]. The relaying node in HARBINGER protocol can be selected from the decoding set in two ways: GeRaF protocol, instantaneous relaying. In $\mathrm{GeRaF}$ protocol the relay closest to the destination will transmit the message whereas in instantaneous relaying the relay with the highest SNR is picked from the decoding set. Multihop transmission can be best explained as a cascade of a point-to-point links where the message is routed to the destination based on a routing algorithm. The throughput analysis and energy efficiency of the aforementioned three protocols are discussed in [8].

A combination dynamic decode forwarding and ARQ for multiple access relay channels with Rayleigh fading is proposed in [28]. A good survey of major ARQ and HARQ contributions is presented in [29]. The range of factors which can affect the performance of the HARQ systems in the presence of cooperative communications and the list of guidelines that can be used while designing a HARQ aided cooperative communication channel are described in [29]. 


\section{Chapter 3}

\section{System Model and Protocol}

This chapter describes the system model and HARQ protocol. The decoding set and other concepts and notations are explained clearly as the chapter proceeds. The parameters of the channel and the concept of outage are discussed. The system simulation is manifested clearly with the help of a flowchart at the end of the chapter.

\subsection{System Model}

The network $\mathcal{N}$ comprises $\mathrm{M}+2$ nodes including a source node $Z_{S}=Z_{M+2}$, a destination node $Z_{D}=Z_{1}$ and $\mathrm{M}$ relays. $Z_{m}$ is the location of node 'm'. Thus, the network may be mathematically expressed as the set

$$
\mathcal{N}=\left\{Z_{m}: 1 \leq m \leq M+2\right\}
$$

The distance between any two nodes is given by

$$
d_{i, j}=\left|Z_{i}-Z_{j}\right|
$$

Relays are numbered in ascending order of their distance to the destination so that

$$
d_{2, D}<d_{3, D}<d_{4, D} \cdots
$$

Every node in the network has a single duplex radio and a single antenna. Time is divided into equal slots. When a node in $\mathcal{N}$ transmits then other nodes should receive. In other 


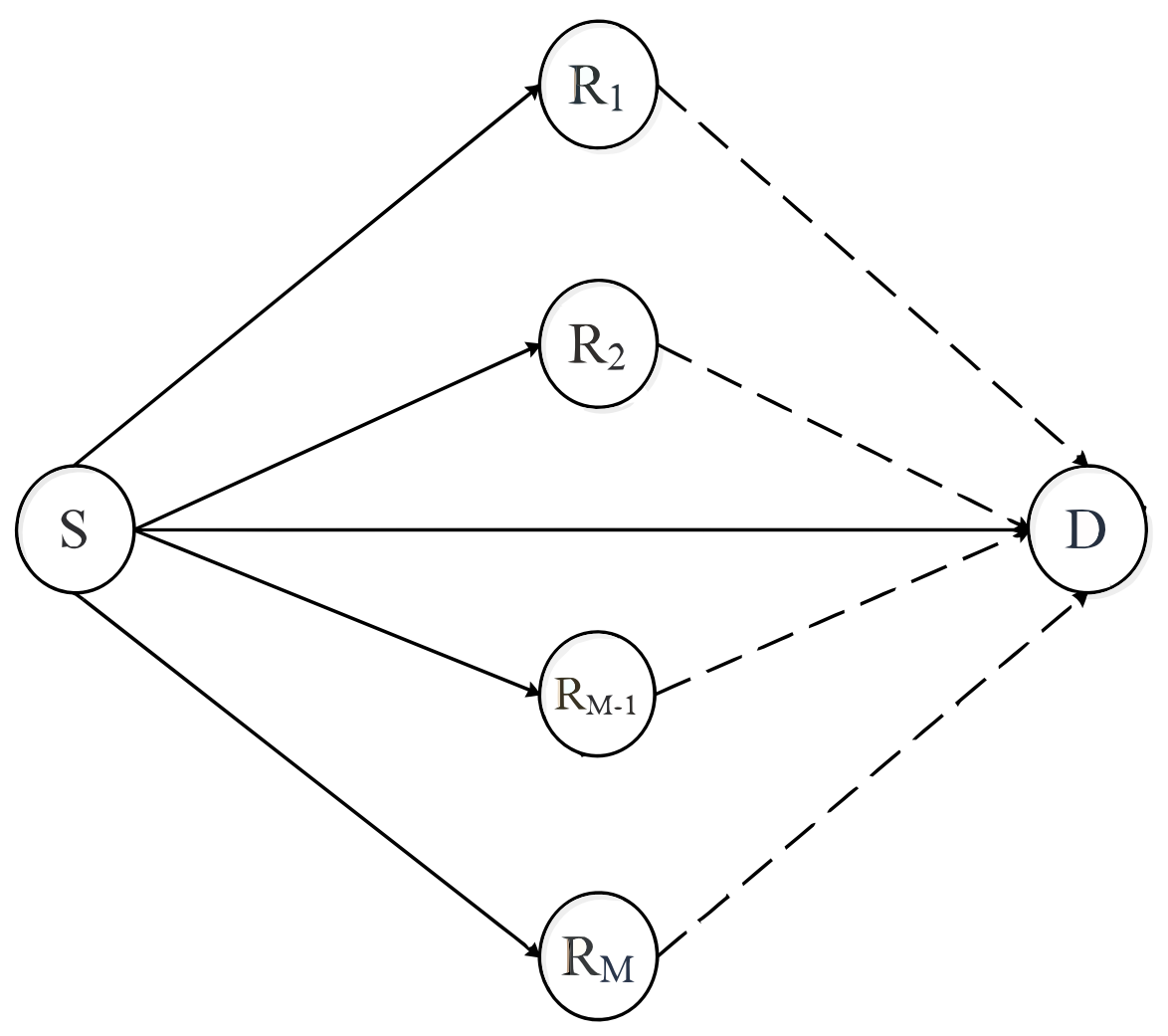

Figure 3.1: A simple M-Relay Network with two hops.

words, a particular node can either transmit or receive in one time slot. It cannot transmit and receive simultaneously.

\section{Decode-and-Forward Strategy}

A simple classic relay channel consists of one relay, but, in general it is assumed that multiple relays are present in between the transmitter and receiver. A simple two hop model with $\mathrm{M}$ relays is shown in Fig. 3.1. As the second transmission can happen from any relay, the links from relays to destination are shown with dotted lines. Decode-and-Forward strategy comes under the category of fixed relaying protocols according to [1]. This thesis follows incremental relaying strategy with decode-and-forward relaying scheme, because after the destination receives the message it replies with ACK/NACK signal, if it is an ACK then re-transmission is not needed. The model described in this thesis considers decoding relays, which are relays that successfully decode the message before forwarding. 


\subsection{Protocol Description}

Conventional wireless networks can be interpreted as a cascade of point-to-point links, and their performance in terms of reliability is poor when the channel fading is severe. The main motive behind choosing cooperative networks is that these algorithms exploit the spatial diversity and the broadcast nature of the relay channel. ARQ protocols have been extensively studied and they are proved to be reliable for data transmission in wireless networks. The thesis studies a decode-forward cooperative Hybrid ARQ relay protocol.

\subsubsection{Hybrid-ARQ}

The hybrid-ARQ protocol reduces the number of re-transmissions than in conventional ARQ scheme and thereby improves the throughput performance of the system. If the receiver cannot successfully decode the message, it discards it and asks for re-transmission. The receiver attempts to decode the re-transmitted code word. In a hybrid-ARQ system the nodes transmit the code word until one of the following occurs: 1) the message has been received successfully and the receiver sends positive acknowledgement (we assume that the feedback channel is error-and-delay-free) 2) the constraint on the maximum number of retransmissions is reached. The code word is re-transmitted only for a limited number of times $N_{\max }$. If the receiver is still not able to correctly decode the code word then it is declared as outage.

\subsubsection{Cooperative Hybrid-ARQ}

Cooperative HARQ combines cooperative communications and HARQ scheme, thereby resulting in a protocol in which re-transmission could come from any node in the network. Hence cooperative HARQ protocol exploits the spatial diversity present in the network.

\section{Decoding Set}

As mentioned [8], it is assumed that any node in the network can estimate its position with onboard global positioning system GPS and it can be assumed that message header has the location coordinates of source and the destination. Hence, nodes in the channel can estimate the average SNR between itself and the destination. 
A new set of channel fading parameters are generated before every signal hop or retransmission. The transmitting node is selected from the decoding set $\mathcal{D}(s)$, the members of the decoding set are called as decoding nodes [8].

Let $\mathcal{D}(s)$ be the set of nodes that have the message at the beginning of a time slot 's'. This $\mathcal{D}(s)$ is called as the decoding set and its members are the decoding nodes. It should be noted that only decoding nodes can re-transmit the signal. The transmitting node in a particular slot is selected from the decoding set, the decision is made by choosing the node that is closest to the destination.

Initially, the decoding set consists of only source and it is updated after every transmission with the nodes that decode the message signal successfully. In the first slot the decoding set consists of only the source node

$$
\mathcal{D}\left(s_{1}\right)=Z_{s}
$$

After a node decodes the message and it is added to the decoding set it is never removed from the decoding set. Therefore, the cardinality of decoding set in current slot is greater than or equal to the cardinality in the previous slot

$$
|\mathcal{D}(s)| \geq|\mathcal{D}(s-1)|
$$

\section{HARBINGER Protocol}

HARBINGER, which stands for hybrid-ARQ-based intracluster geographic relaying [8], is a generalization of the hybrid-ARQ protocol. As mentioned earlier, the relays could also retransmit the message, provided it has decoded the earlier transmitted blocks. The protocol hence provides the spatial diversity effect and this diversity is achieved without requiring the relays to co-phase their transmissions. A similar assumption is made for the protocol implemented in this thesis. The comparison of HARBINGER with multihop relaying is explained in [8].

\section{GeRaF Protocol}

GeRaF stands for Geographic Random Forwarding. It is a transmission scheme based on geographical routing where the node that acts as the relay is not known by the sender, but rather is decided after the transmission has taken place. The key idea behind GeRaF 
protocol [9] is that node transmits the information packet that composes of its own location and the location of the destination. The nodes that receive the packet will try act as a relay, and the one that serves as a relay is based on how close it is to the destination.

\section{Comparison of HARBINGER, GeRaF and Protocol Implemented}

While the protocol used in this thesis is similar to GeRaF and HARBINGER protocols, there is a subtle difference. In GeRaF protocol described in [9] once a node is selected from the decoding set all other nodes in the network clear their memory of the message, but this protocol assumes that the nodes retain the message information. In HARBINGER the relays and destination can combine the information sent by the source and other relays, this contributes to the transmit diversity. For the protocol described in thesis, the node that is selected from the decoding set only broadcasts the signal to the destination. The protocol described in this thesis uses Type-I HARQ. Whereas, HARBINGER uses Type-II HARQ protocol. Type-I HARQ is chosen to reduce the complexity in the system while deriving of the closed form expression for outage and throughput.

\subsection{Channel parameters}

\subsubsection{Path Loss Exponent}

According to the inverse power law the power of the signal falls off with the inverse of the square of distance. The received power when expressed in terms of the transmitted power is attenuated by a factor called as path loss exponent $\alpha$. Power decays quadratically with distance in free space [17]. On ground, path loss is found to take on values between 2.5 to 4 .

\subsubsection{Rayleigh Fading}

Fading is the random fluctuation of the received signal power about its mean value and it is attributed to factors such as reflection, diffraction and scattering [30]. Fading is inevitable in wireless networks and many fading models exist that try to model the power distribution in a wireless channel. In this thesis, we assume that the wireless channel to have small scale fading that follows Rayleigh distribution. When the fading amplitude is a Rayleigh random variable, the received power, which is the square of the amplitude, can be assumed to follow 
a Rayleigh distribution. The probability density function (p.d.f) of an exponential random variable $X$ is given by

$$
f_{X}(x)=\frac{1}{\lambda} \exp \left(-\frac{x}{\lambda}\right) \quad \text { for } \quad x \geq 0 .
$$

where $\lambda$ is the expected value or mean of exponential random variable. The mean of $X$ is given by

$$
E[X]=\Gamma d^{-\alpha} .
$$

where $d$ is distance of separation between the transmitter and receiver, $\Gamma$ is Signal to Noise Ratio (SNR) at unit distance and $\alpha$ is path loss exponent.

\subsubsection{Outage Threshold and Probability}

Outage threshold is the minimum value of the Signal to Noise Ratio below which outage occurs. Outage threshold denoted by $\beta$ though it is often expressed in $\mathrm{dB}$.

In decibels

$$
\beta(d B)=\log _{10} \beta .
$$

The rate is related to outage threshold by

$$
\text { Rate }=\log _{2}(1+\beta) .
$$

The signal to noise ratio $\gamma$ at the receiving node should be greater than outage threshold $\beta$, so that it could be decoded and retransmitted to the receiver. The outage threshold is the maximum limit for SNR below which outage occurs. The probability that outage occurs is termed outage probability $P_{\text {out }}$, and given by

$$
P_{\text {out }}=\mathrm{P}[\gamma \leq \beta] .
$$


The outage probability is the cumulative distribution function of (3.5)

$$
P_{\text {out }}=1-\exp \left(\frac{-\beta}{\lambda}\right) .
$$

\subsection{Simulation}

The protocol described Section 3.2.1, is simulated using MATLAB. The code for simulation is constructed using the algorithm in fig.3.2. Owing to the dynamic nature of the channel, fading parameters are generated in the beginning of every transmission cycle. As it is mentioned earlier in section 3.3.2, a Rayleigh fading model is considered. For each link, a random variable corresponding to the SNR is drawn according to the distribution of that link. The value of random SNR is compared to the outage threshold. If it is below the outage threshold, an outage is declared, otherwise, it is assumed the code word is successfully decoded. A decoding set is maintained that is updated after each transmission cycle. The decoding set consists of all the nodes that decoded the message successfully. By default, the decoding set comprises the source, and when a node is added to decoding set it is never removed from the set.

After updating the decoding set, the closest node to the receiver is selected and that node acts as the source in the next transmission. If the closest node is the destination itself then the transmission is successful. Otherwise the process continues for a predefined number of times before the limit on maximum number of transmissions is reached $\left(N_{\max }\right)$. 


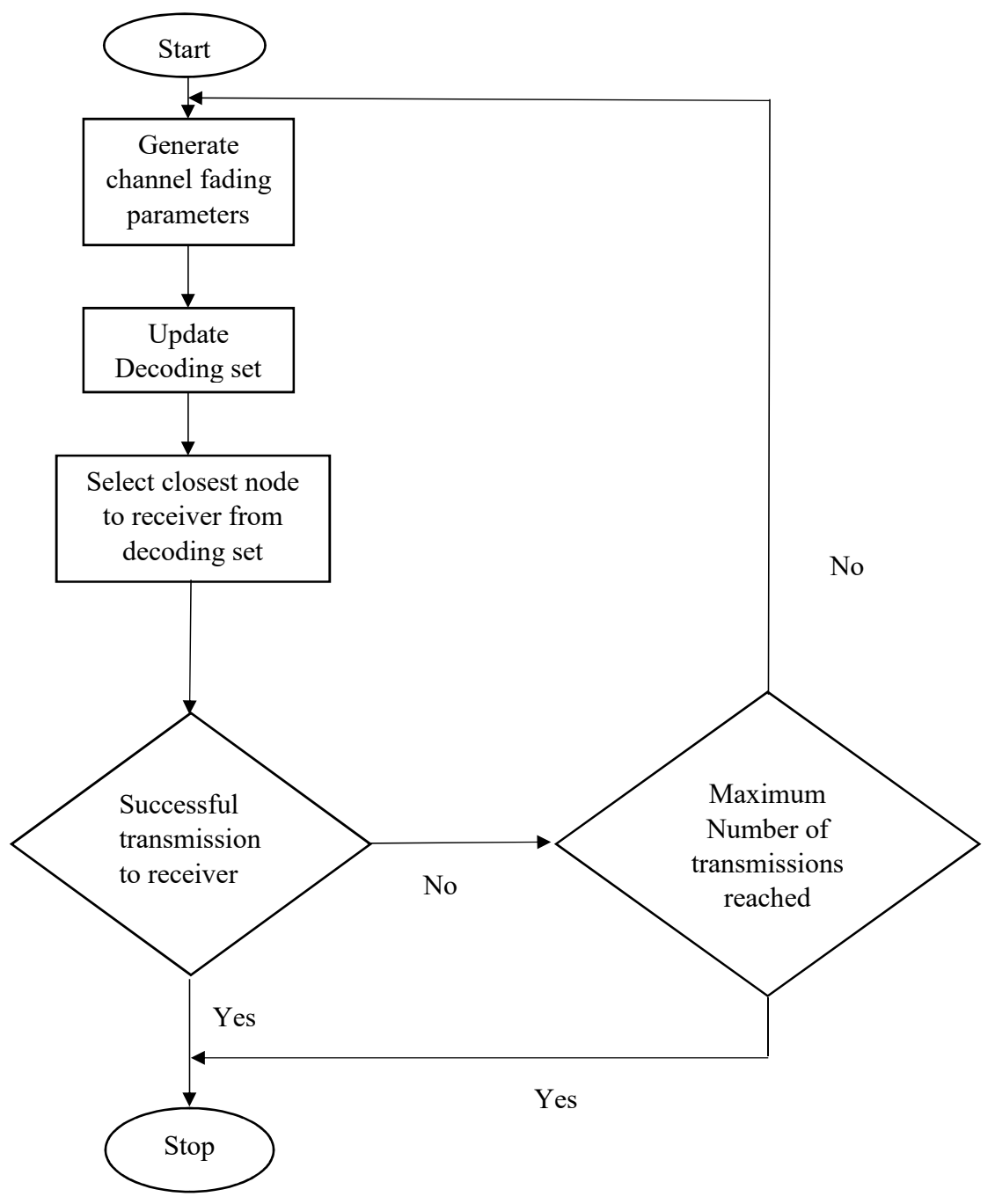

Figure 3.2: Flowchart for simulation. 


\section{Chapter 4}

\section{Throughput Analysis of Fixed Networks}

This chapter analyzes the protocol described earlier in an step by step procedure. The problem is disintegrated into three cases: when there are no relays, one relay and general case when there are 'n' number of relays.

In fixed networks the location of the nodes is preset. This type of configuration is especially helpful to analyze the network performance precisely. Line topologies are ideal examples of fixed networks. In line networks relays are located on the straight line joining transmitter and receiver. For simplicity we assume that the nodes are separated by equal distances. A simple line topology is illustrated in Fig. 4.1.

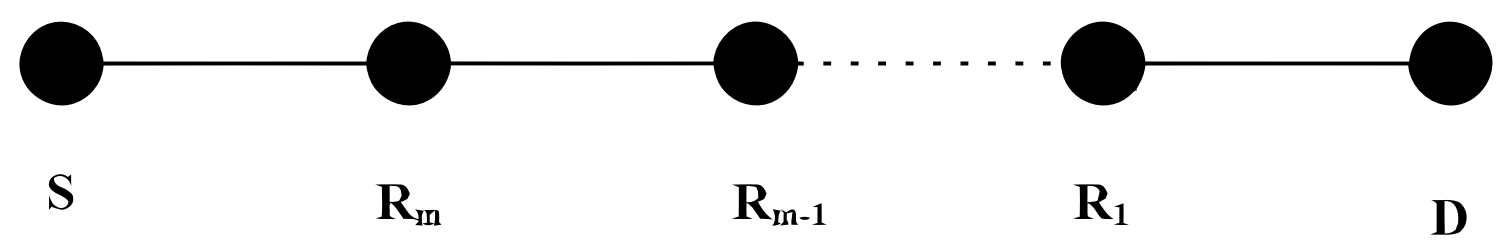

Figure 4.1: Simple line network 


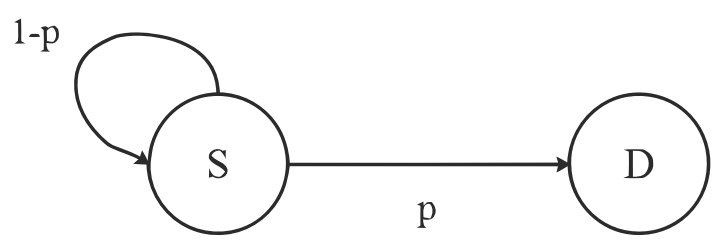

Figure 4.2: Markov chain for no relays case.

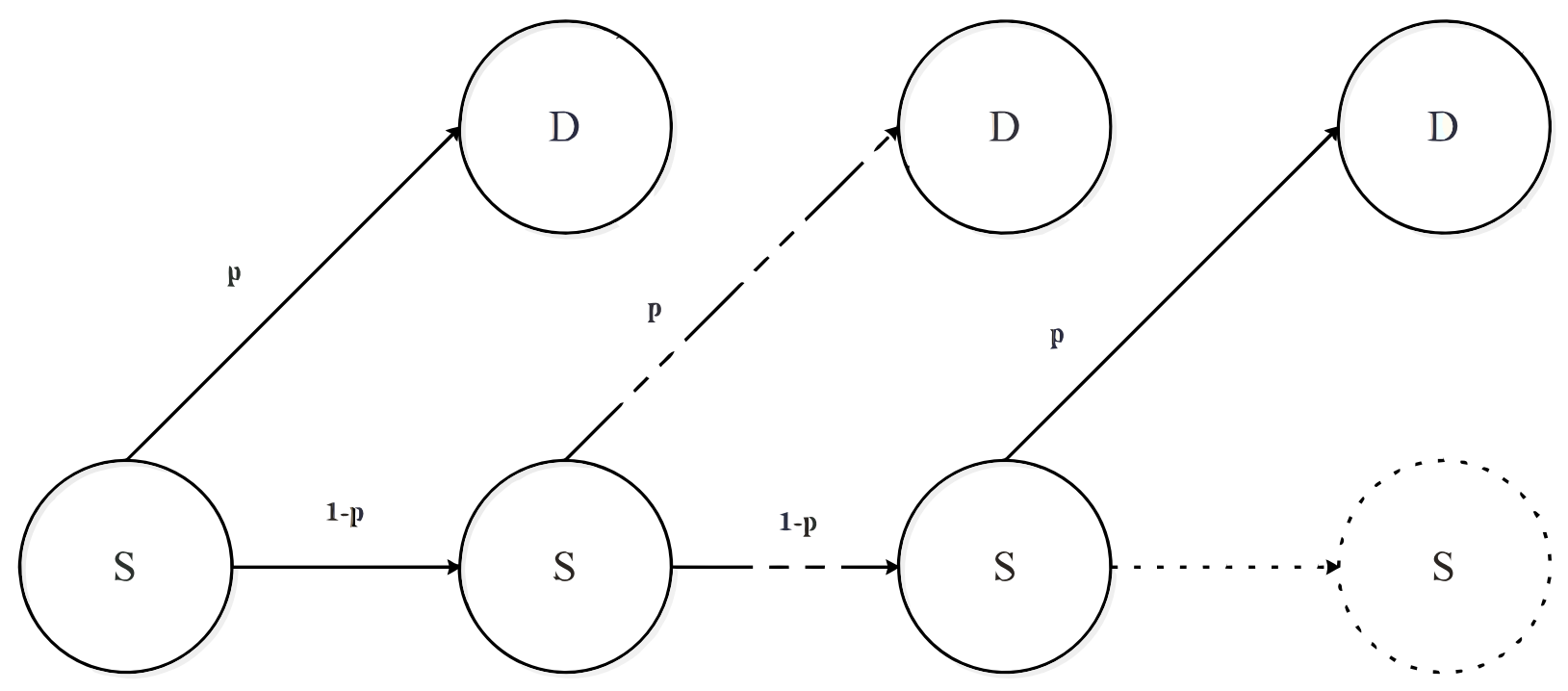

Figure 4.3: Trellis diagram for no relays case

\subsection{Analysis of Line Networks Using Markov Chains}

The line network is analyzed in an incremental procedure with the help of Markov chains in this section. Three cases are explained with the help of Markov chains and trellis diagrams.

\subsubsection{No Relays}

A simple case of network realization is studied, when there are no relays between the transmitter and receiver. In other words this is scenario when a direct link is being established. It is best represented with the help of Markov chain shown in Fig. 4.2. Markov chain is a mathematical model for stochastic processes. There are two Markov states possible: successful transmission and unsuccessful transmission. The transition probabilities from one state to other are indicated in the Fig: 4.3. The probability of a successful transmission 
is $p$ and failure is $1-p$. The probability of success $p$ is given by

$$
p=\exp \left(\frac{-\beta}{\lambda_{S D}}\right) .
$$

A trellis diagram can be generated from Markov chain, shows the passage of time. In a successful transmission the signal ends up at one of the destination nodes, where as, in an unsuccessful transmission the signal ends up in dotted nodes. The trellis diagram for no relays case is shown in Fig. 4.3. The probability of success after every hop can be represented with a geometric RV given by

$$
P[n]= \begin{cases}p & \text { for } n=1 \\ p(1-p) & \text { for } n=2 \\ p(1-p)^{N_{\max }-1} & \text { for } n=N_{\text {max }}\end{cases}
$$

$N_{\max }$ is the maximum number of transmissions allowed before it is counted as failure. The above case is called as truncated H-ARQ because the number of re-transmissions are restricted to $N_{\max }$. The random variable in (4.2) is a truncated geometric random variable.

\subsubsection{One Relay}

The next step is to introduce one relay to the previous case, and the purpose of the relay is to cooperate with the signal transmission. The Markov chain and the trellis diagram shown in Fig. 4.4 and Fig. 4.5 are used to obtain the probability mass function of success after every hop. Several rules are observed while using Markov chains.

- The sum of the branches coming out of any node should be equal to unity.

- The probability that a particular node has successfully received the message is the sum of weight of all the paths that meet at the node

- The values of the matrix in the top row, corresponding to D in Fig. 4.5 show the probabilities of success after the $n^{\text {th }}$ hop.

- The message should finally end up at destination or at one of the dotted nodes in the last hop shown in Fig. 4.5. The dotted nodes correspond to the outage states, and the 


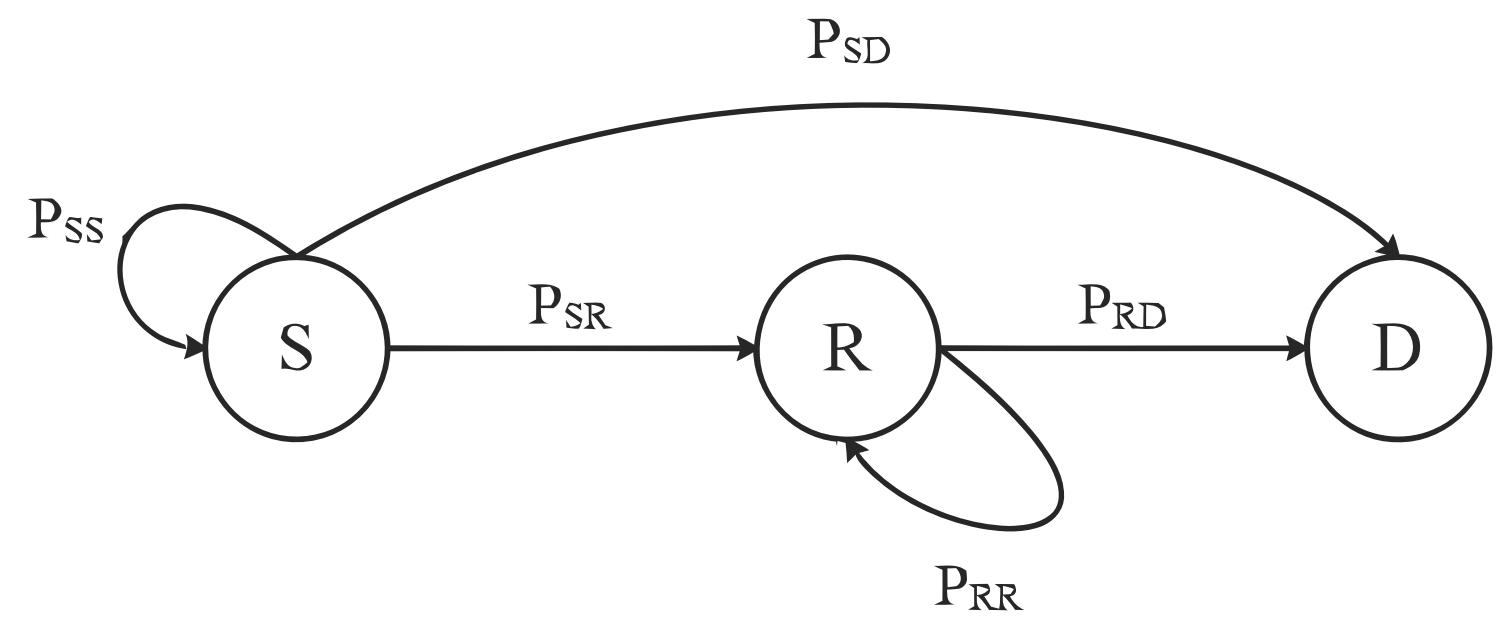

Figure 4.4: Markov Chain for one relay.

sum of the probabilities is the outage probability.

- The states of the nodes are denoted as $\mathrm{S}(\mathrm{i}), \mathrm{R}(\mathrm{i})$ and $\mathrm{D}(\mathrm{i})$ in the trellis diagram Fig.

4.5. These correspond to the probability that the chain is in that state at time $i$.

\section{Link Probabilities}

The link probabilities between any two nodes are of five types

1. Link from source to destination

$$
\begin{aligned}
P_{S D} & =P\left[\gamma_{S D}>\beta\right] . \\
& =\exp \left(\frac{-\beta}{\lambda_{S D}}\right) .
\end{aligned}
$$

2. Link from source to relay

$$
\begin{aligned}
P_{S R} & =P\left[\gamma_{S R}>\beta\right] P\left[\gamma_{S D} \leq \beta\right] \\
& =\exp \left(\frac{-\beta}{\lambda_{S R}}\right)\left(1-\exp \left(\frac{-\beta}{\lambda_{S D}}\right)\right) .
\end{aligned}
$$




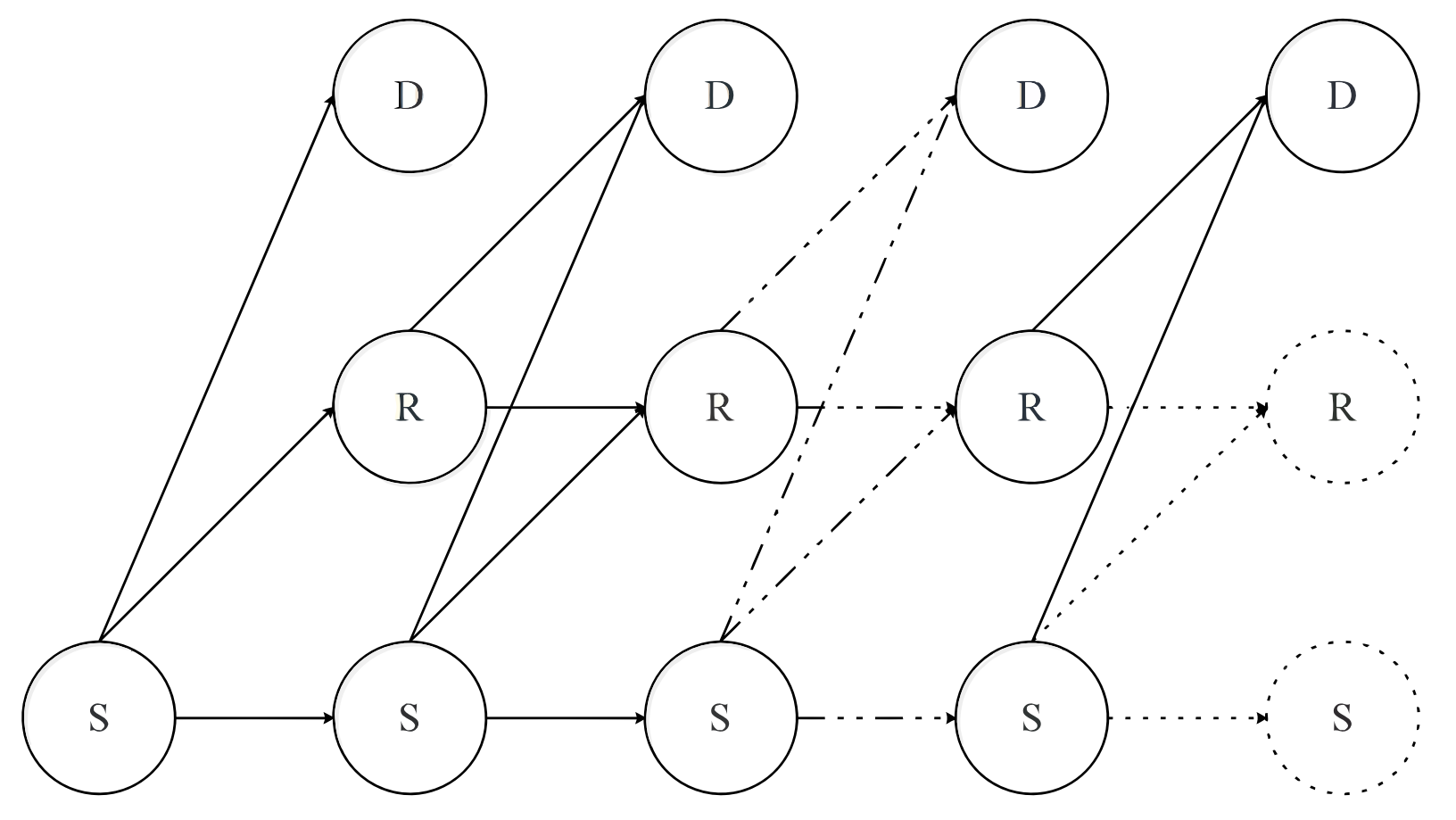

Figure 4.5: Trellis diagram for one relay.

3. Link from relay to destination

$$
\begin{aligned}
P_{R D} & =P\left[\gamma_{R D}>\beta\right] \\
& =\exp \left(\frac{-\beta}{\lambda_{R D}} .\right)
\end{aligned}
$$

4. When the transmission from source fails and none of the nodes are able to decode the source transmission then the weight of link from source to itself is

$$
\begin{aligned}
P_{S S} & =1-P_{S R}-P_{S D} \\
& =1-\exp \left(\frac{-\beta}{\lambda_{S R}}\right)\left(1-\exp \left(\frac{-\beta}{\lambda_{S D}}\right)\right)-\exp \left(\frac{-\beta}{\lambda_{S D}}\right) .
\end{aligned}
$$

5. When the transmission from a relay node fails then the weight of link from the relay to itself is

$$
\begin{aligned}
P_{R R} & =1-P_{R D} \\
& =1-\exp \left(\frac{-\beta}{\lambda_{R D}}\right) .
\end{aligned}
$$


The procedure to obtain pmf's from the Markov chain is described with the help of example below.

Example: The procedure to find the probability of success of link in third hop is described. There are three possible paths over which the signal can end up at the destination.

$$
\begin{aligned}
& S \stackrel{1}{\rightarrow} R \stackrel{2}{\rightarrow} R \stackrel{3}{\rightarrow} D \\
& S \stackrel{1}{\rightarrow} S \stackrel{2}{\rightarrow} R \stackrel{3}{\rightarrow} D \\
& S \stackrel{1}{\rightarrow} S \stackrel{2}{\rightarrow} S \stackrel{3}{\rightarrow} D
\end{aligned}
$$

We calculate the weight of every path and sum them up to find the probability of success in third hop.

\section{Finding the node probabilities}

Source always has the message

$$
\begin{gathered}
S(0)=1 . \\
S(1)=P_{S S} . \\
S(2)=S(1) P_{S S} . \\
S(3)=S(2) P_{S S} .
\end{gathered}
$$

Relay node in first hop

$$
R(1)=S(0) P_{S R} .
$$

Relay node in second hop

$$
R(2)=S(1) P_{S R}+R(1) P_{R R}\left(1-P_{R D}\right) .
$$




$$
\begin{gathered}
D(3)=R(2) P_{R D}+S(2) P_{S D} . \\
P[3]=D(3)=P_{p a t h 1}+P_{p a t h 2}+P_{p a t h 3} .
\end{gathered}
$$

\subsubsection{Multiple Relays}

The next step of getting closer to practicality is to implement the case of multiple relays. The Markov chain and trellis diagram for multiple relays case are shown in 4.6 and 4.7 respectively. The procedure followed for multiple relays case is identical to one relay case, except that there is increase in the number of branches flowing into and out of the nodes. As the number of relays goes on increasing it gets difficult to represent the process in the form of diagram. An algorithm that can be used to compute the probability mass functions is given below.

\section{Generic algorithm for determining the probability mass functions}

1. For computational flexibility the trellis diagram can be represented in form of matrix. The state of the nodes in every hop is indicated with a separate entry in the matrix, resulting in a matrix with $M+2$ rows and $N_{\max }$ columns.

$$
\left[\begin{array}{cccc}
D(1) & D(2) & \ldots & D\left(N_{\max }\right) \\
R_{1}(1) & R_{1}(2) & \ldots & R\left(N_{\max }\right) \\
\cdot & & & \\
\cdot & & & \\
R_{M}(1) & R_{M}(2) & \ldots & R_{M}\left(N_{\max }\right) \\
S(1) & S(2) & \ldots & S\left(N_{\max }\right)
\end{array}\right]
$$

2. The computation of the matrix elements is done column by column. The computation of the first column is easy and straight forward because there are no previous states, as it is the first transmission.

3. The computation of next $N_{\max }-1$ columns is done in an iterative method. The conditional probability of a node successfully decoding the signal is dependent on the factor that other nodes that are closer to the destination fail in decoding the message. 


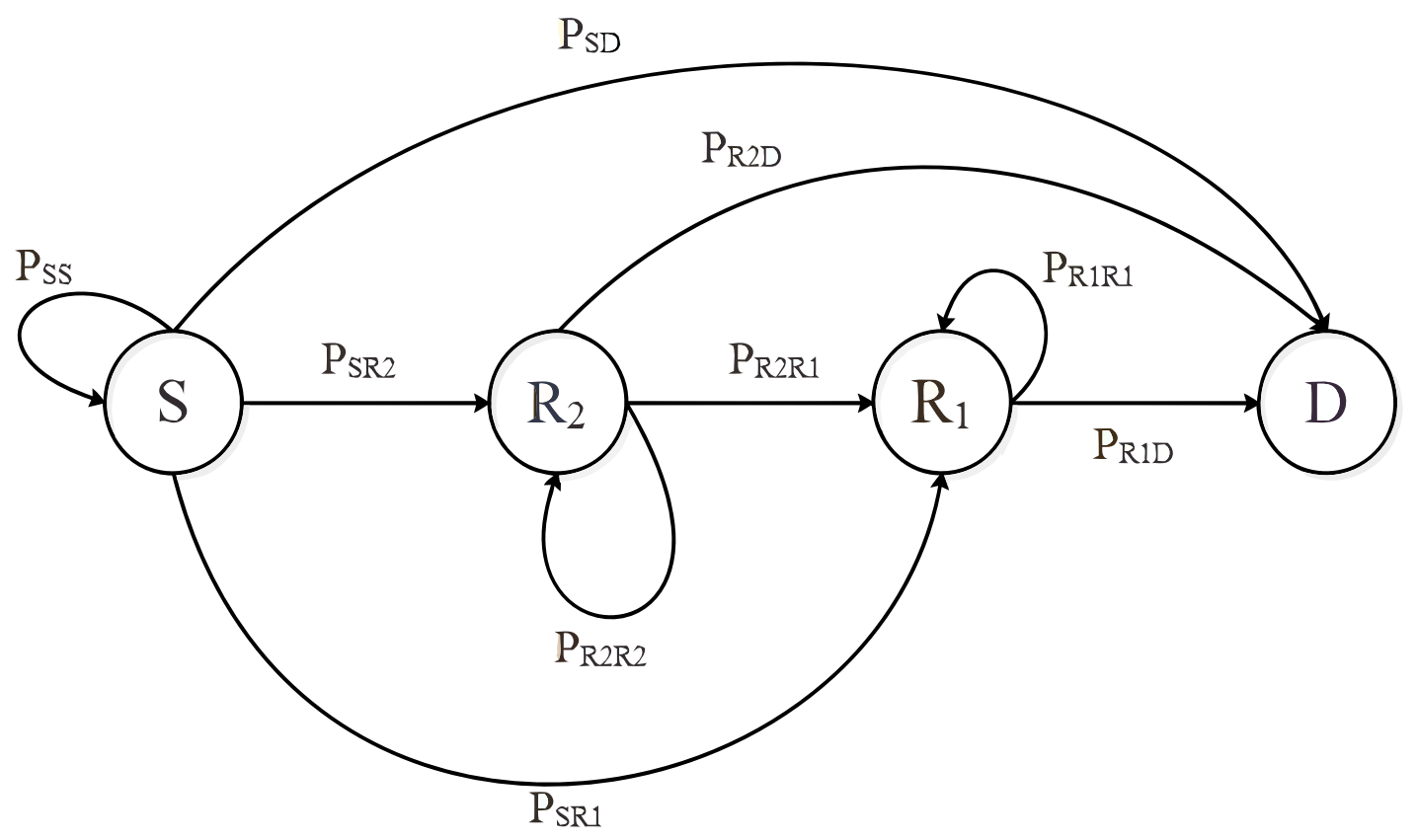

Figure 4.6: Markov chain for multiple relays case.

4. The last column of the matrix is the final hop and it is directly the sum of all the weighted branches flowing into the node.

5. The dotted nodes in the trellis diagram shown in fig. 4.7 are failure states, they do not necessarily need to be computed as they are result in outage.

6. The end result of matrix computation leads to the probabilities of successes for a particular number of hops. These probability mass functions are significant as they are required to compute the outage, throughput and effective throughput.

The probability mass function pmf for $N$ with constraint on number of re-transmissions $N_{\max }$ is given by the following equation

$$
P[n]= \begin{cases}D(1) & \text { for } n=1 \\ D(2) & \text { for } n=2 \\ D\left(N_{\max }\right) & \text { for } n=N_{\max }\end{cases}
$$




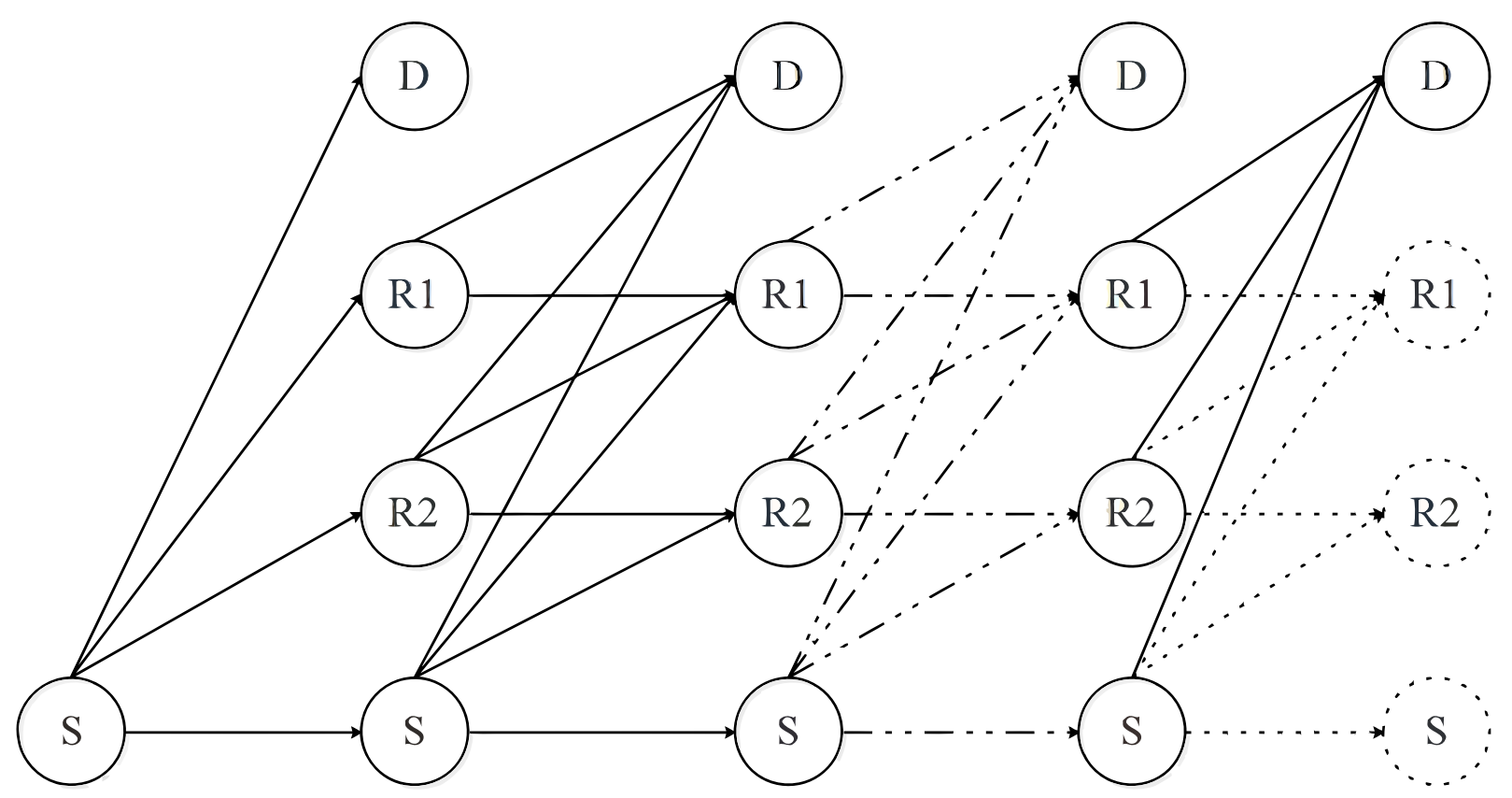

Figure 4.7: Trellis diagram for multiple relays.

\subsection{Outage Probability and Effective Throughput}

The closed form expressions for outage, throughput and effective throughput are derived in this section. The closed form expressions obtained are implemented for fixed network topologies and results are discussed.

\subsubsection{Outage Probability}

Outage probability is defined as the probability that signal is not correctly decoded at the destination. It can be determined from the trellis diagram

$$
\begin{aligned}
P_{\text {out }} & =1-P(\text { success }) \\
& =1-\sum_{n=1}^{N_{\max }} P[n] .
\end{aligned}
$$

\subsubsection{Throughput and Effective Throughput}

Throughput ' $\eta$ ' is defined as the average amount of information that has been successfully transmitted in one time slot. Throughput can be obtained from pmf of $\mathrm{N}$ with constraint 
$N_{\max }$ on the number of re-transmissions in (4.15)

$$
\begin{aligned}
P_{\text {success }} & =P\left[N \leq N_{\max }\right] \\
& =\sum_{n=1}^{N_{\max }} P[n] .
\end{aligned}
$$

First, pmf is scaled by normalization factor $\xi$ to make it a valid pmf [31]

$$
\begin{gathered}
\xi=\left[\sum_{n=1}^{N_{\max }} P[n]\right]^{-1} . \\
P_{0}[n]=\frac{P[n]}{\sum_{n=1}^{N_{\max }} P[n]} .
\end{gathered}
$$

The mean of total number of re-transmissions given success is

$$
E[N \mid \text { success }]=\sum_{n=1}^{N_{\max }} n P_{0}[n] .
$$

Throughput $\eta$ is the average amount of information that has been transmitted assuming that the transmissions are successful

$$
\begin{aligned}
\eta & =\frac{\log _{2}(1+\beta)}{E[N \mid \text { success }]} \\
& =\frac{\beta}{\sum_{n=1}^{N_{\max }} n P_{o}[n]} .
\end{aligned}
$$

Effective throughput $\eta_{e f f}$ can be defined as the data that has been successfully transmitted

$$
\eta_{\text {eff }}=\frac{P_{\text {success }} \log _{2}(1+\beta)}{E[N \mid \text { success }]} .
$$

\subsubsection{Numerical results}

This section presents the effective throughput and outage performance of the system under the impact of various parameters.

\section{Parameter selection}


Before generating the results, the fading parameters used to model the system are set. Unless specified that a parameter is changing they are set to the default values that are given below.

- Number of relays $M=9$

- Path loss exponent $\alpha=3.5$

- SNR at unit distance from the transmitter $\Gamma=30 \mathrm{~dB}$

- Distance of separation between the transmitter and the receiver $r_{\text {net }}=10$

Three sets of results are generated

1. Variation of $P_{\text {out }}$ and $\eta_{\text {eff }}$ with number of relays: It is observed that the effective throughput increases with the increase in number of relays and outage performance of the system also improves with higher number of relays. Outage performance of the network and the effective throughput curves are shown in Fig. 4.8 and Fig. 4.9.

2. Impact of path loss exponent on $P_{\text {out }}$ and $\eta_{\text {eff }}$ : In general, a high path loss exponent reduces the system throughput as shown in Fig. 4.13 and outage performance is also degraded as in Fig. 4.10. The effect of number of relays on the throughput performance of the system is studied when the medium fading is varies from a minimal value of 2 to a maximum of 4 .

3. Impact of $S N R \Gamma$ on $P_{\text {out }}$ and $\eta_{\text {eff }}$ : Increase in $\Gamma$ positively effects the system outage performance shown in Fig. 4.12. 


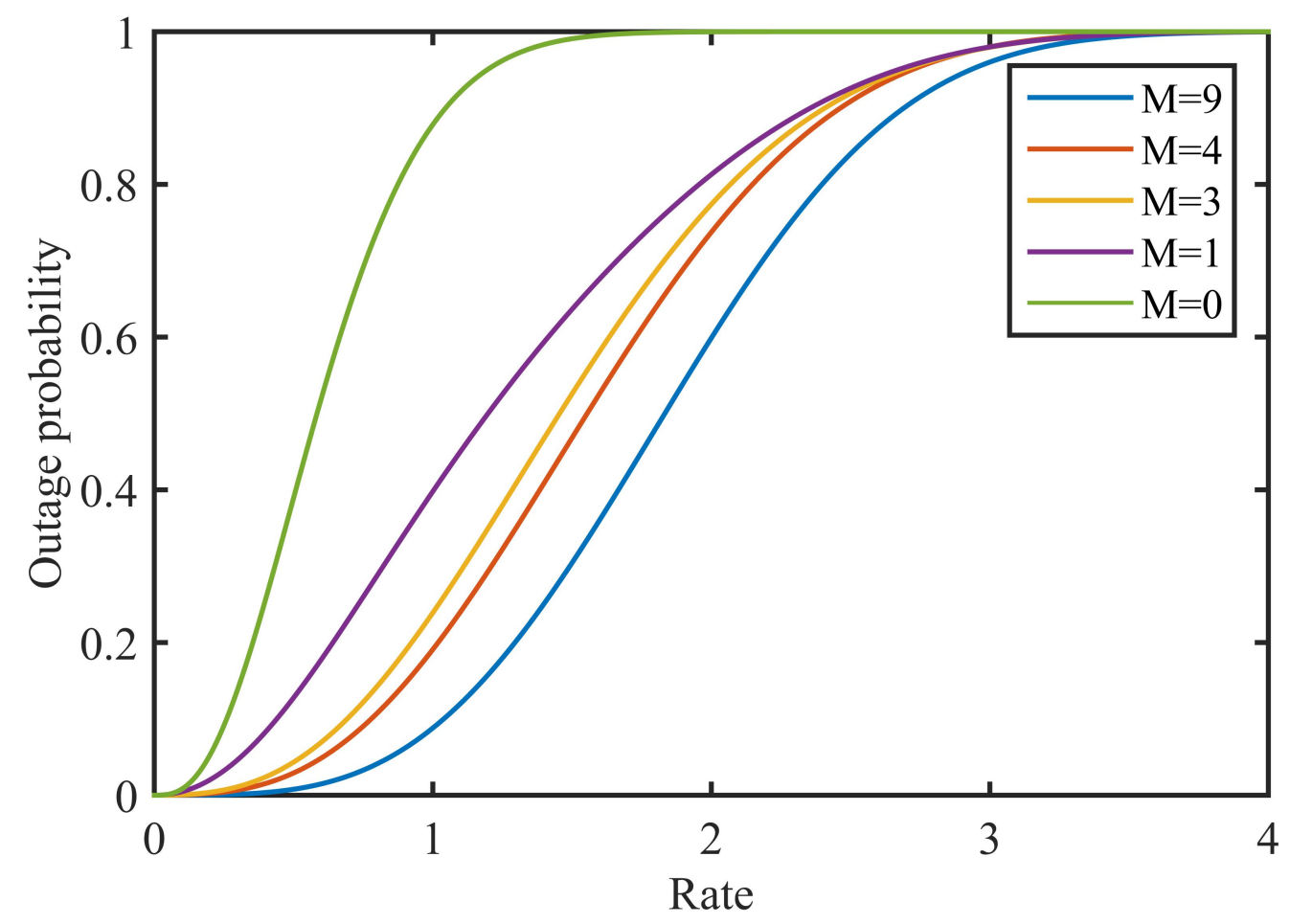

Figure 4.8: $P_{\text {out }}$ for the change in $\mathrm{M}$ when $\alpha=3.5$ and $\Gamma=30 \mathrm{~dB}$.

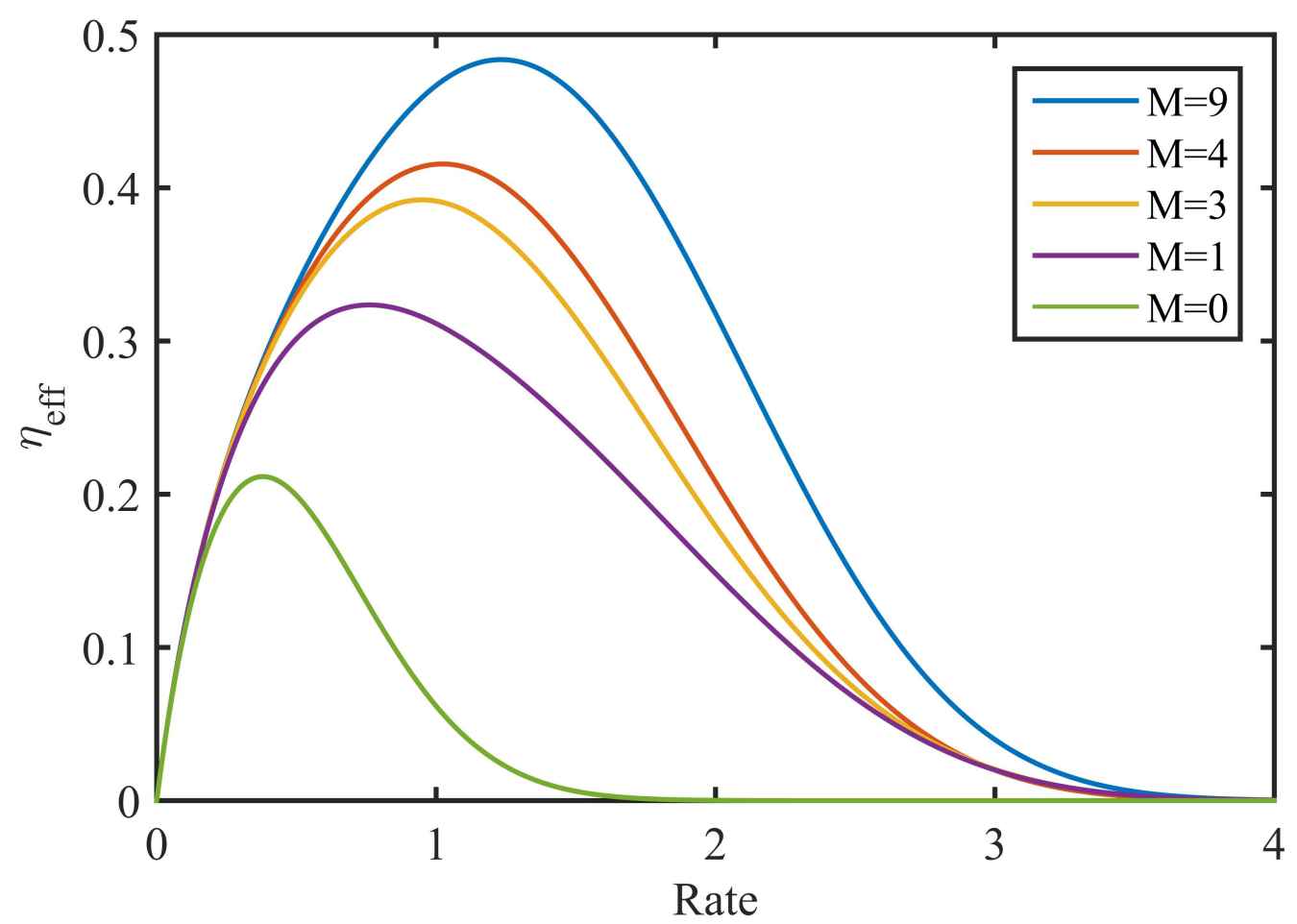

Figure 4.9: Effective throughput for change in $\mathrm{M}$ when $\alpha=3.5$ and $\Gamma=30 \mathrm{~dB}$. 


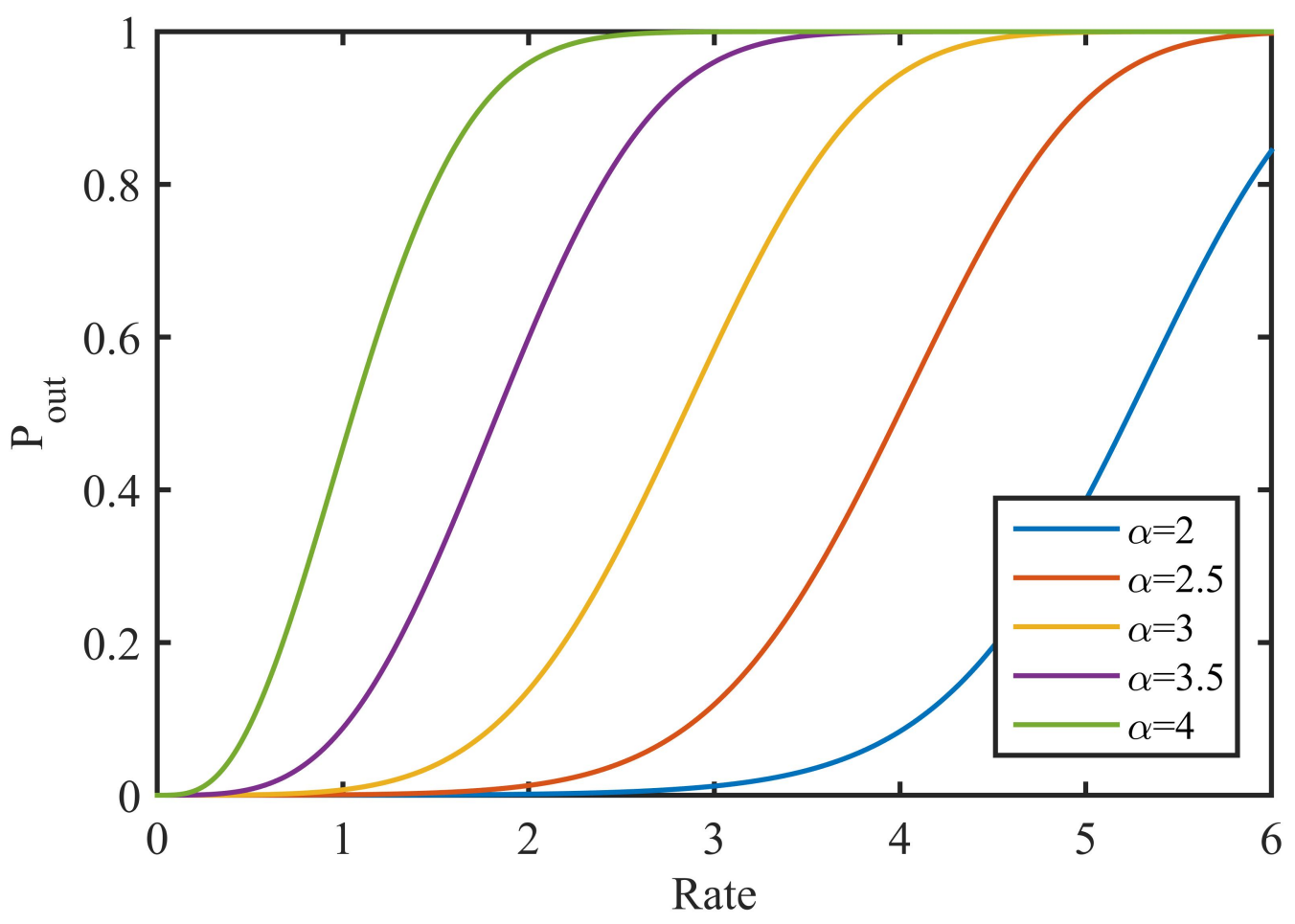

Figure 4.10: Impact of $\alpha$ on $P_{\text {out }}$ for $M=9$ and $\Gamma=30 \mathrm{~dB}$.

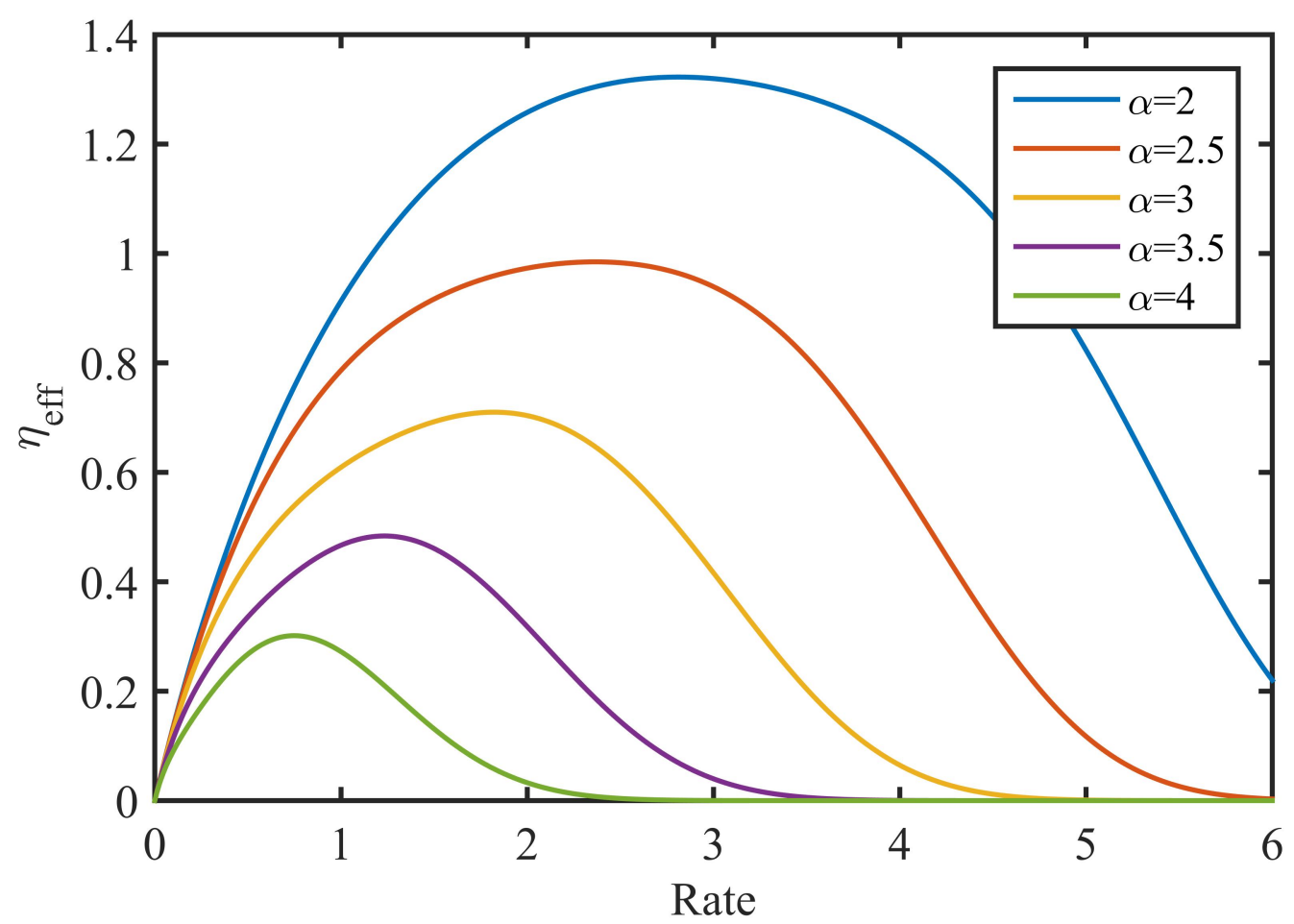

Figure 4.11: Impact of $\alpha$ on $\eta_{\text {eff }}$ for $M=9$ and $\Gamma=30 \mathrm{~dB}$. 


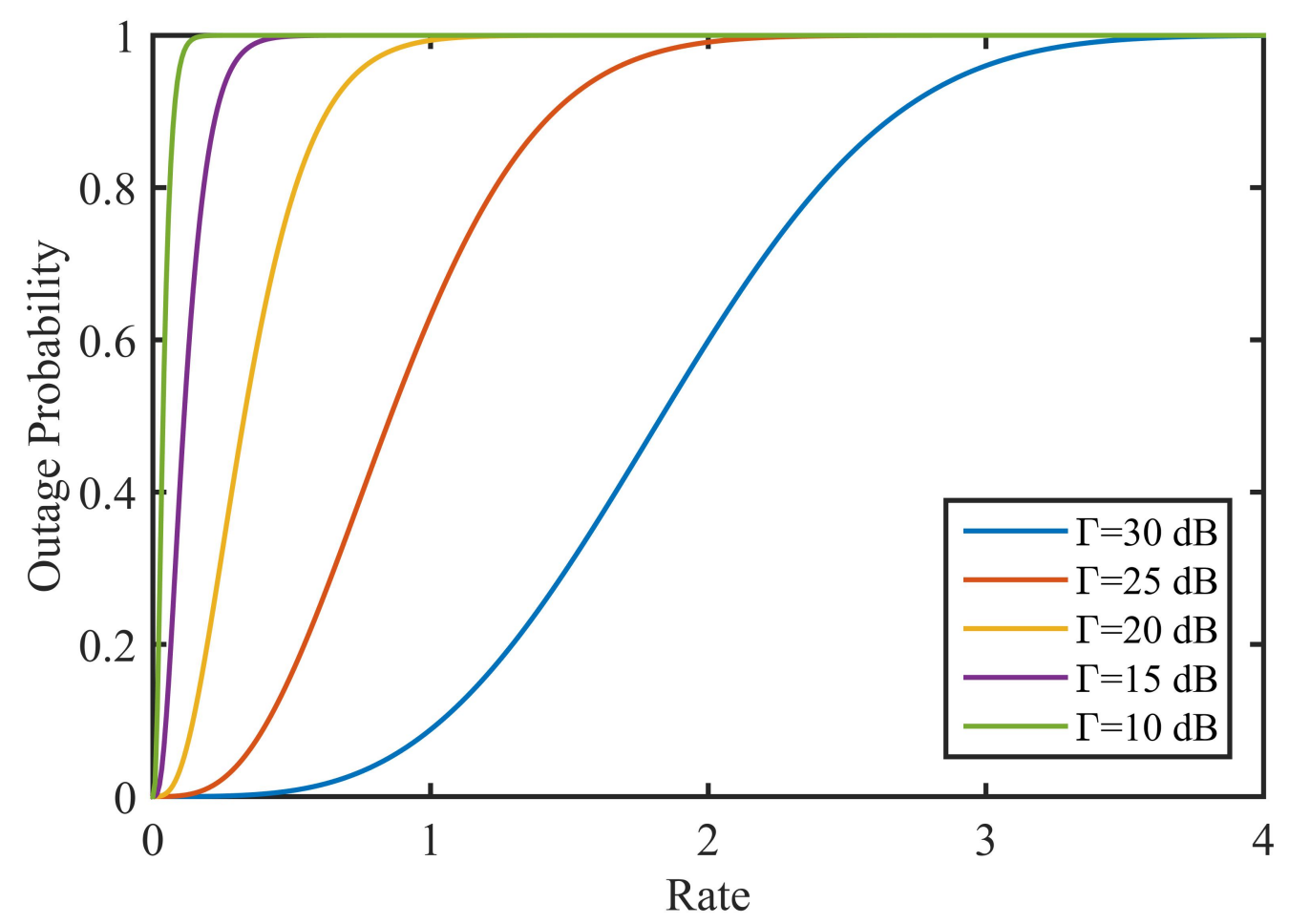

Figure 4.12: Impact of $\Gamma$ on $P_{\text {out }}$ for $M=9$ and $\alpha=3.5$.

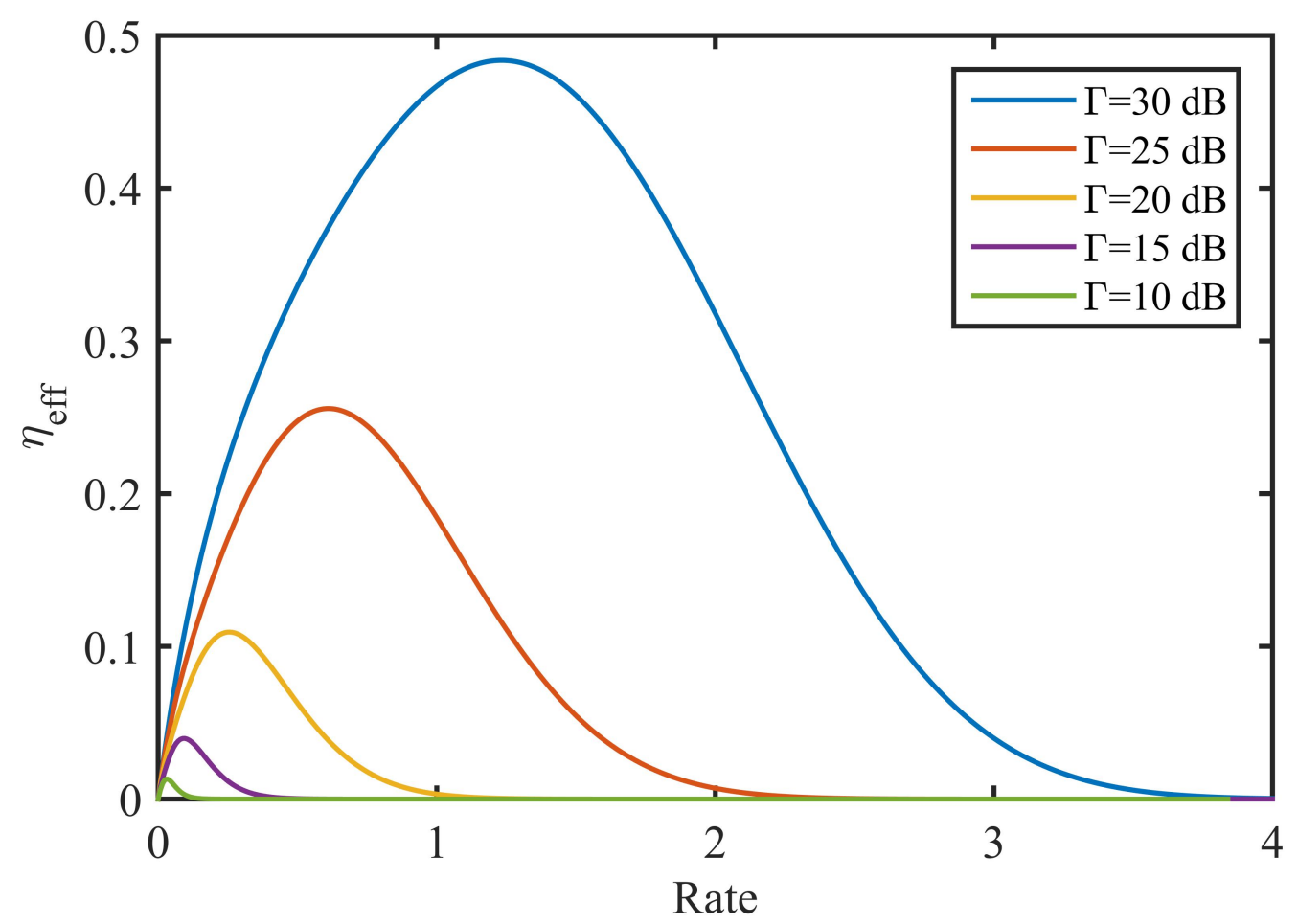

Figure 4.13: Impact of $\Gamma$ on $\eta_{\text {eff }}$ for $M=9$ and $\alpha=3.5$. 


\section{Chapter 5}

\section{Spatial Averaging and Random Networks}

This chapter considers random network topologies. The performance metrics such as the outage probability and effective throughput, which are explained in the previous chapter, are computed for random topologies. Due to the random placement of the relays and as the performance of protocol is sensitive to the topology, it is necessary to make sure that spatial variations of the system are being accounted for. Hence the later section of the chapter deals with the spatial averaging as a BPP and PPP.

\subsection{Random Networks}

This model assumes that relays are independent and uniformly distributed (i.u.d) over the entire plane $\mathbb{R}^{2}$. The source transmitter and receiver locations are fixed. While analyzing the network we consider that the relays with in an imaginary circular boundary are able to capture the transmission more effectively than the relays located outside the boundary. The circle borderline is assumed to have receiver at its center and transmitter located on the boundary. The network is shown in Fig. 5.1. Although the transmitter broadcasts the message signal and every relay in the vicinity of the transmitter receives the message, only the relays that lie within the circular boundary are best suited to cooperate with the signal transmission. The prime motive behind choosing the relays that lie with in the boundary is, they are close to the receiver and within the transmission range of the source. 


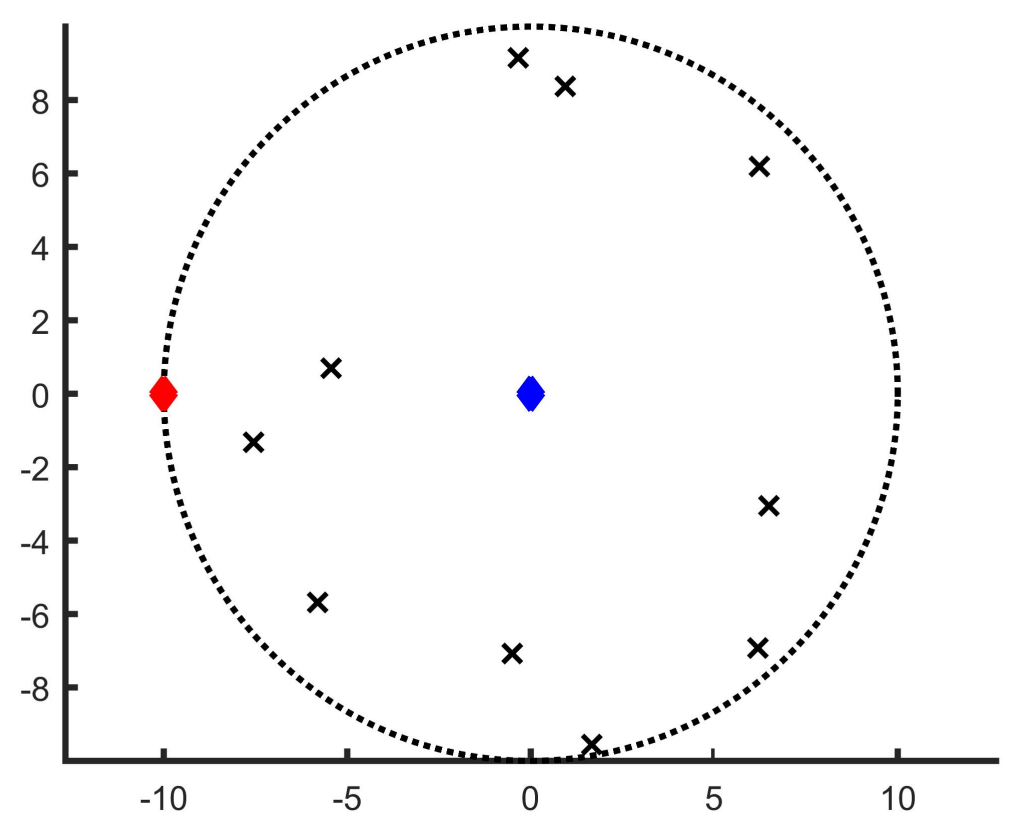

Figure 5.1: Random network model.

\subsection{Effect of Network Topology on System Performance}

In the previous chapter on fixed topologies, the location of the nodes in the system is fixed. While considering the case of random networks the position of the nodes changes from one network realization to the other. The change in the position of the nodes from one hop to the other changes the distances between the nodes. This random placement of the relays influences the network parameters significantly. Hence it is important to identify that the system performance is dependent on network topology and same is shown in Fig. and Fig.

The analysis of random network is similar to the analysis of fixed networks mentioned in the previous chapter. Markov chain model is used to deduce the outage probability and effective throughput of the system. The expressions for outage probability and effective throughput mentioned in the previous chapter are implemented to this random model.

\subsection{Spatial Average}

Spatial configuration of the network is important in deciding the performance of the network. The geographical disposition of relays is significant because distance is critical parameter in deciding the signal power. A system cannot be conceived for a single spatial 


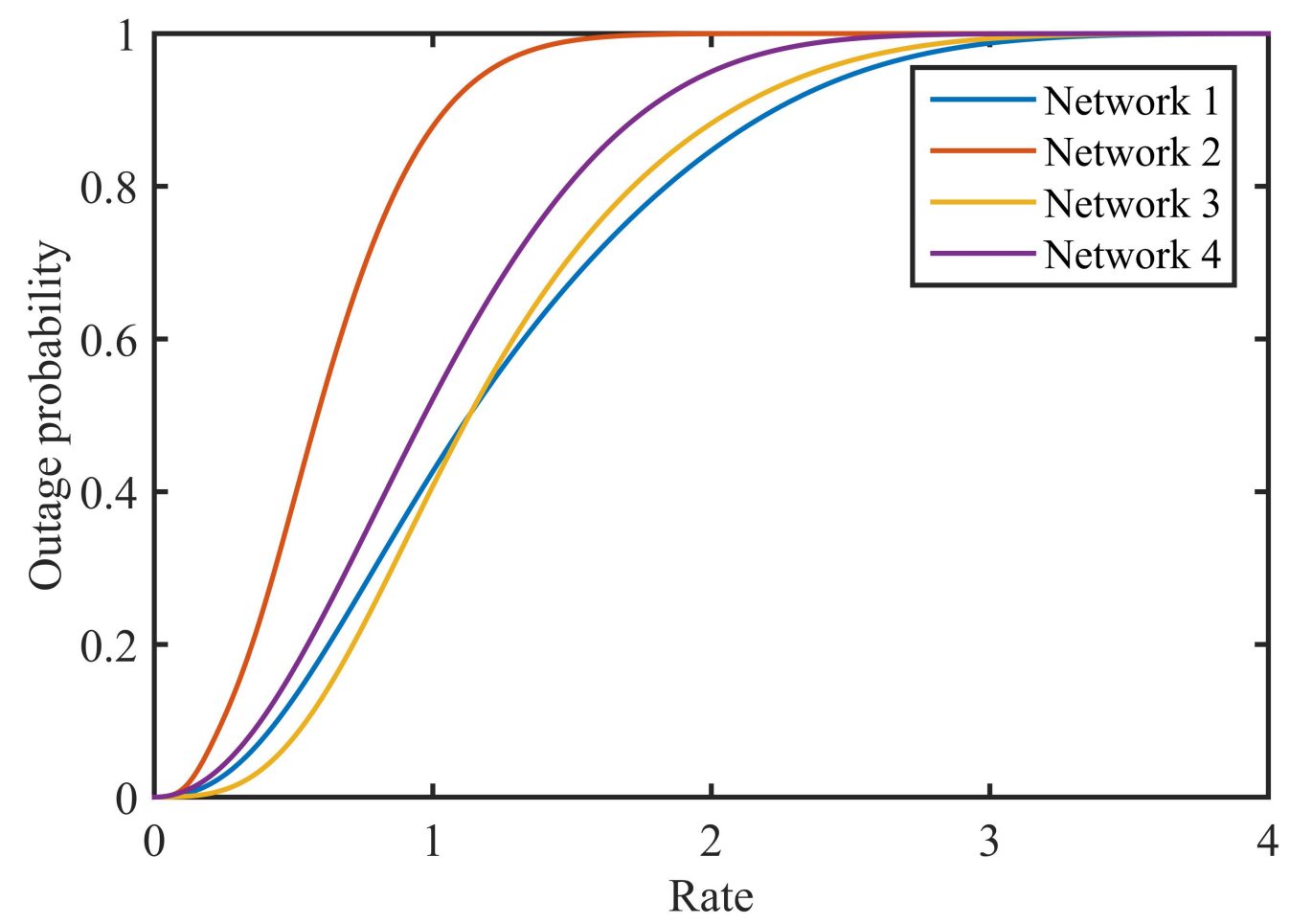

Figure 5.2: Effect of random placement of relays on $P_{\text {out }}$.

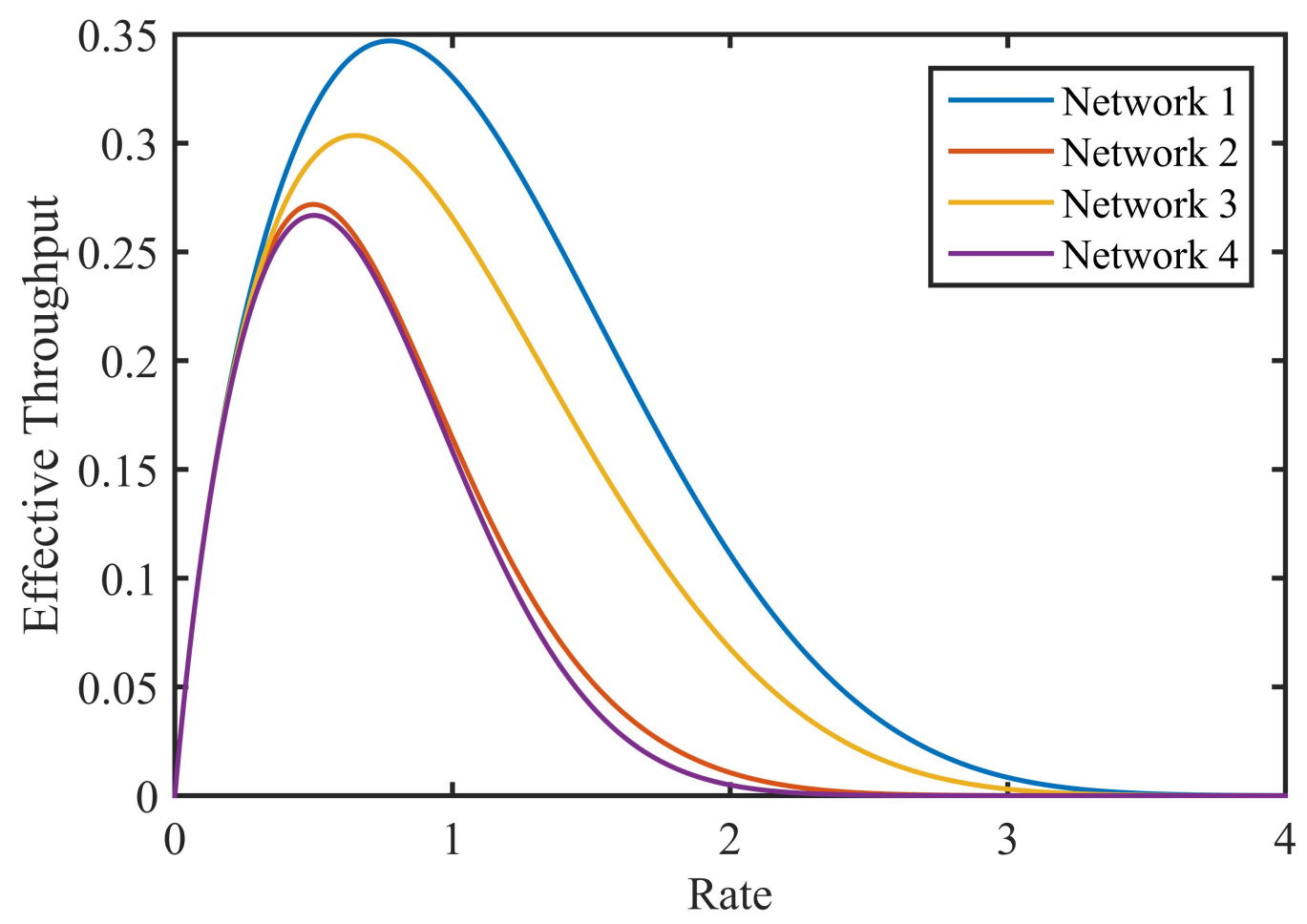

Figure 5.3: Effect of random placement of relays on $\eta_{\text {eff }}$. 
configuration because various configurations are possible. This leads to finding out a metric that not only captures the fading effects present but also the spatial variations of the system. Spatial averaging is an effective way to estimate the performance of the system. A direct approach to spatial averaging is adopted [10].

\subsubsection{Spatial Averaging when the Network is BPP defined over Region}

When the relays are independently and uniformly distributed i.u.d then they are said to be drawn from a binomial point process.

- Initially, the number of relays $\mathrm{M}$ is fixed.

- After setting up M, Monte Carlo simulation of $\mathrm{N}$ networks is generated, with each network consisting of M relays

- Outage probability and effective throughput are computed for every Monte Carlo trial after network generation and they are accumulated in an array

- The obtained outage probability and effective throughput are averaged with respect to number of network topologies generated

- The spatial averaging is done for all values of $\mathrm{M}$ and it is denoted as $\epsilon[M]$.

The spatially averaged outage probability and effective throughput for BPP are shown in the results section of this chapter.

\subsubsection{Poisson Point Process}

The direct approach to determine the PPP outage probability and effective throughput is adopted [10].

- First, BPP spatial averages are obtained

- The intensity $\Lambda$ of PPP on the plane $\mathbb{R}^{2}$ is defined as

$$
\Lambda=\frac{E[M]}{|\mathcal{A}|} .
$$

where $|\mathcal{A}|$ is the area of the region 
- The poisson mean with $\mathrm{M}$ relays within region $\mathcal{A}$ is given by

$$
\mathbb{E}[M]=\Lambda|\mathcal{A}|
$$

- The probability mass function of the number of relays within $\mathcal{A}$ is given by [18]

$$
p_{M}[m]=\frac{(\Lambda|\mathcal{A}|)^{m}}{m !} \exp (-\Lambda|\mathcal{A}|), \quad \text { for } m \geq 0 .
$$

- The spatially averaged outage in PPP is found by taking expectation of spatially averaged outage (BPP) with respect to the number of relays.

$$
\epsilon=\sum_{m=0}^{\infty} p_{M}[m] \epsilon[m] .
$$

- As it is not practically possible to compute an infinte summation. The sum in (5.4) is computed only upto specific limit of $\mathrm{m}$, say $M_{\max }$. The value of $M_{\max }$ is chosen such that $\epsilon \rightarrow 1$. Truncated summation of spatially averaged outage is given by

$$
\epsilon=\sum_{m=0}^{M_{\max }} p_{M}[m] \epsilon[m] .
$$

\subsubsection{Numerical Results}

This section presents the spatially averaged effective throughput and outage performance of the system under the impact of various parameters.

Parameter selection Before generating the results, the fading parameters used to model the system are set. Unless specified that a parameter is changing they are set to the default values given below.

- Number of network realizations generated $=10000$

- Number of relays $M=10$

- Path loss exponent $\alpha=3.5$

- SNR at unit distance from the transmitter $\Gamma=30 d B$ 
- Distance of separation between the transmitter and the receiver $r_{n e t}=10$

Three sets of results are generated

1. Variation of $P_{\text {out }}$ and $\eta_{\text {eff }}$ with number of relays: It is observed that the effective throughput increases with the increase in number of relays and outage performance of the system also improves with higher number of relays. Outage performance of the network and the effective throughput curves are shown in Fig. 4.8 and Fig. 4.9.

2. Impact of path loss exponent on $P_{\text {out }}$ and $\eta_{\text {eff }}$ : In general, a high path loss exponent reduces the system throughput as shown in fig. 4.13 and outage performance is also degraded as in Fig. 4.10. The effect of number of relays on the throughput performance of the system is studied when the medium fading is varies from a minimal value of 2 to a maximum of 4 .

3. Impact of $S N R \Gamma$ on $P_{\text {out }}$ and $\eta_{\text {eff }}$ : Increase in $\Gamma$ positively effects the system outage performance shown in Fig. 4.12.

4. Performance metrics for PPP The outage probability and the effective throughput of the system when the relays placement is random and when they are drawn from a poisson distribution are shown in Fig. 5.10 and Fig. 5.11.

5. Cumulative distribution function of maximum throughput: The cumulative distribution function indicates the percentage of network realizations that attain maximum throughput less than a specified value. CDF curves are plotted in MATLAB using a special function called as "ecdf". The CDF curve for BPP and PPP are shown in Fig. 5.12 and 5.13 . 


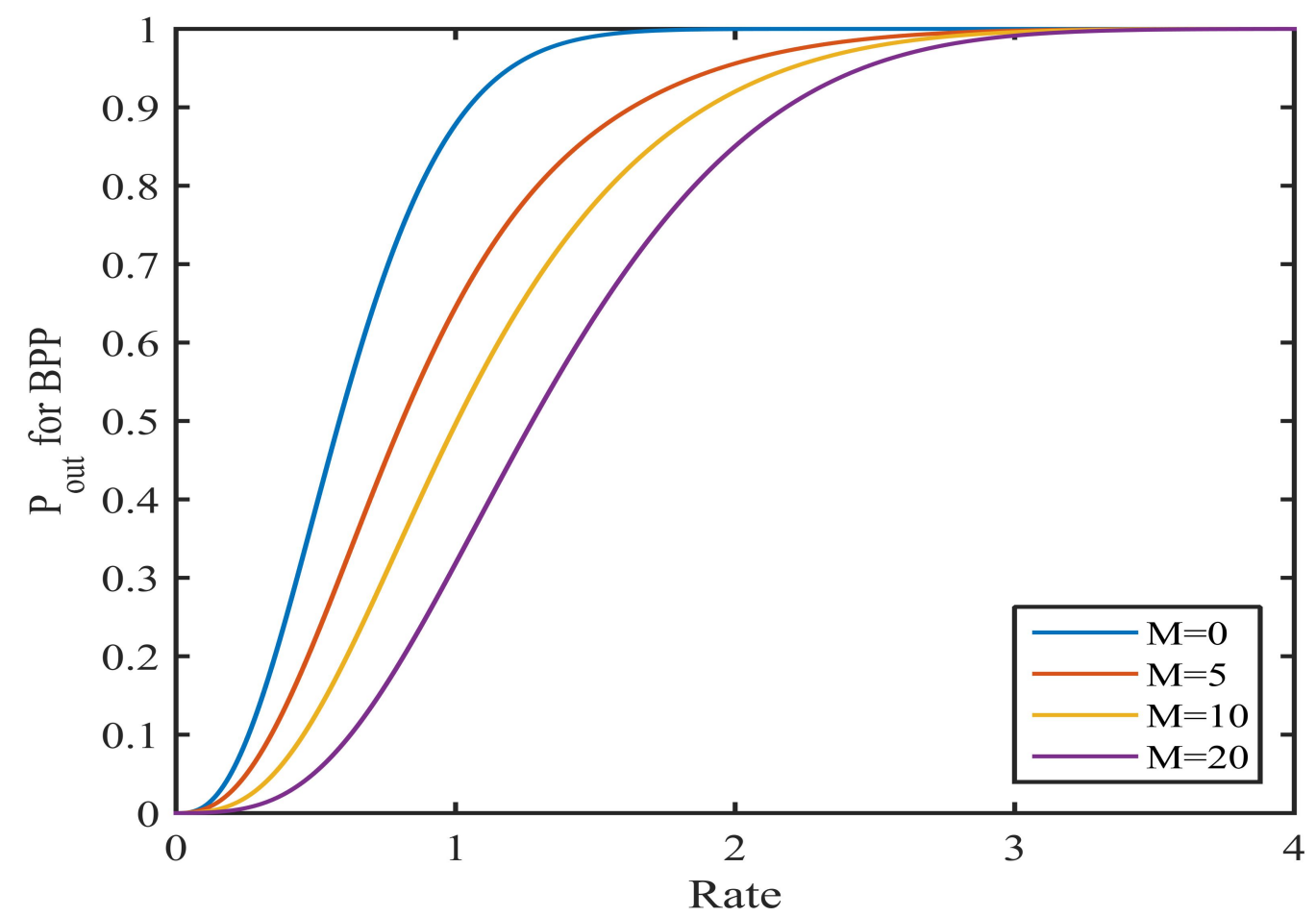

Figure 5.4: Spatially averaged outage probability for $\Gamma=30 \mathrm{~dB}$ and $\alpha=3.5$.

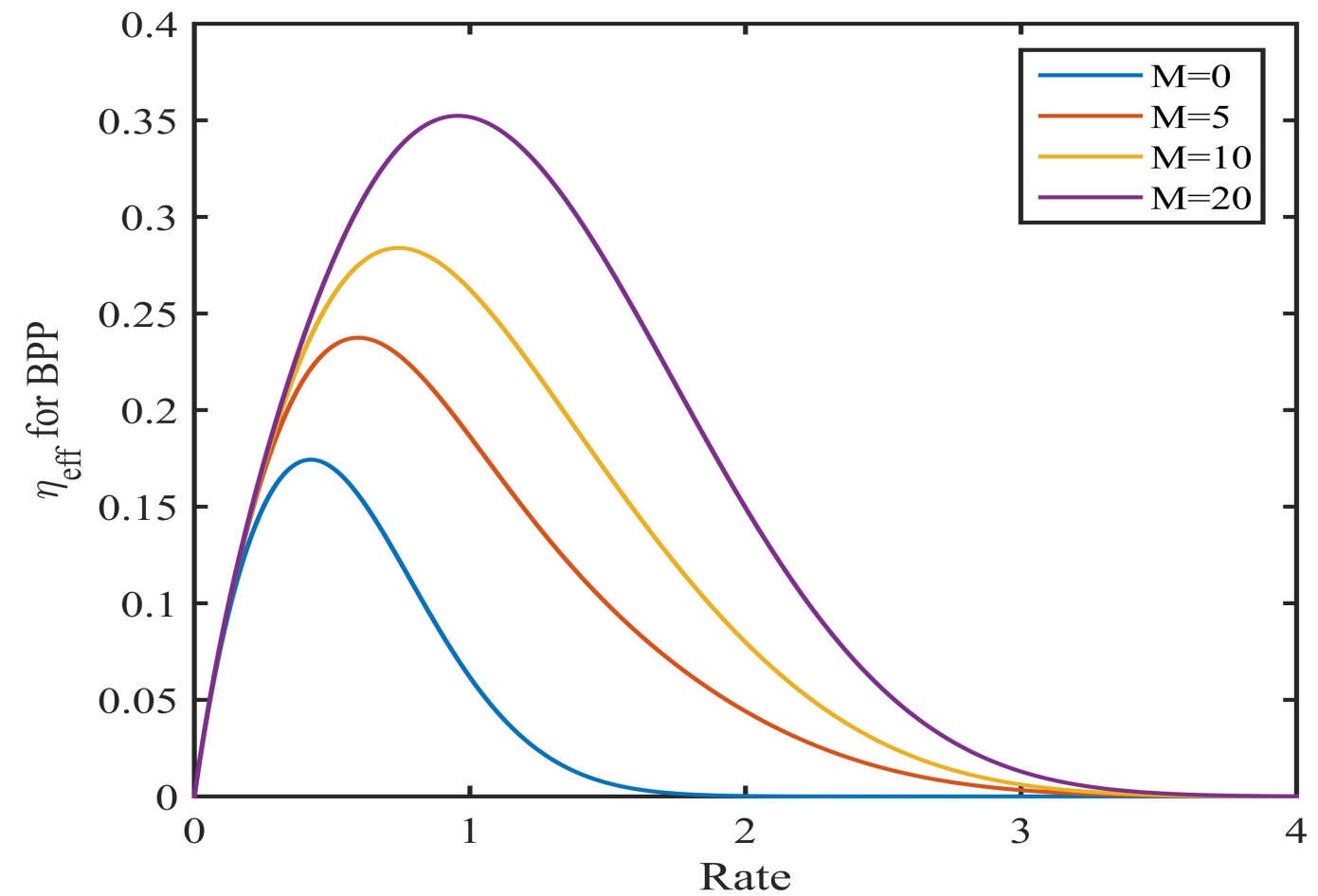

Figure 5.5: Spatially averaged effective throughput for $\Gamma=30 \mathrm{~dB}$ and $\alpha=3.5$. 


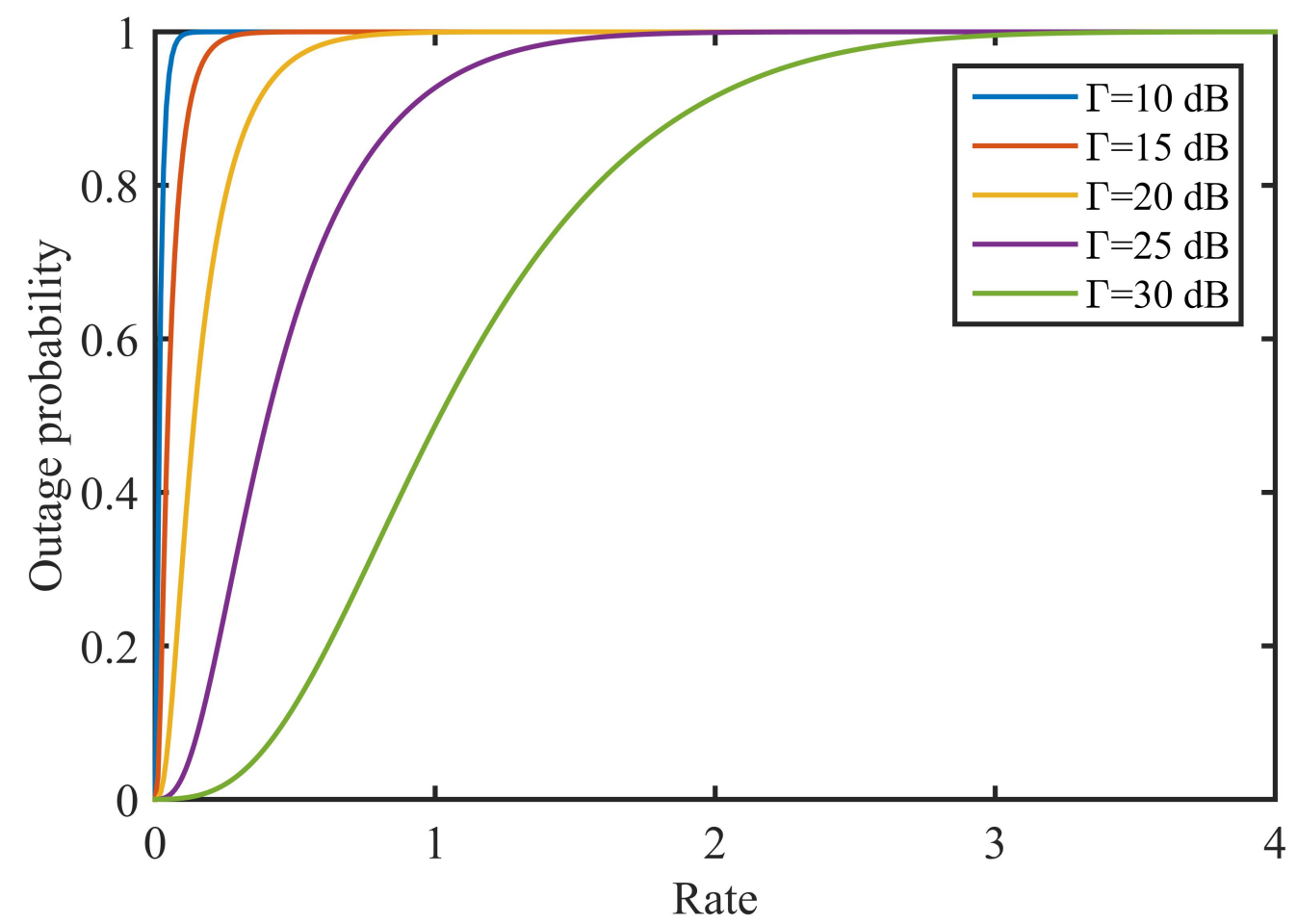

Figure 5.6: Spatially averaged outage probability for $\alpha=3.5$ and $M=10$.

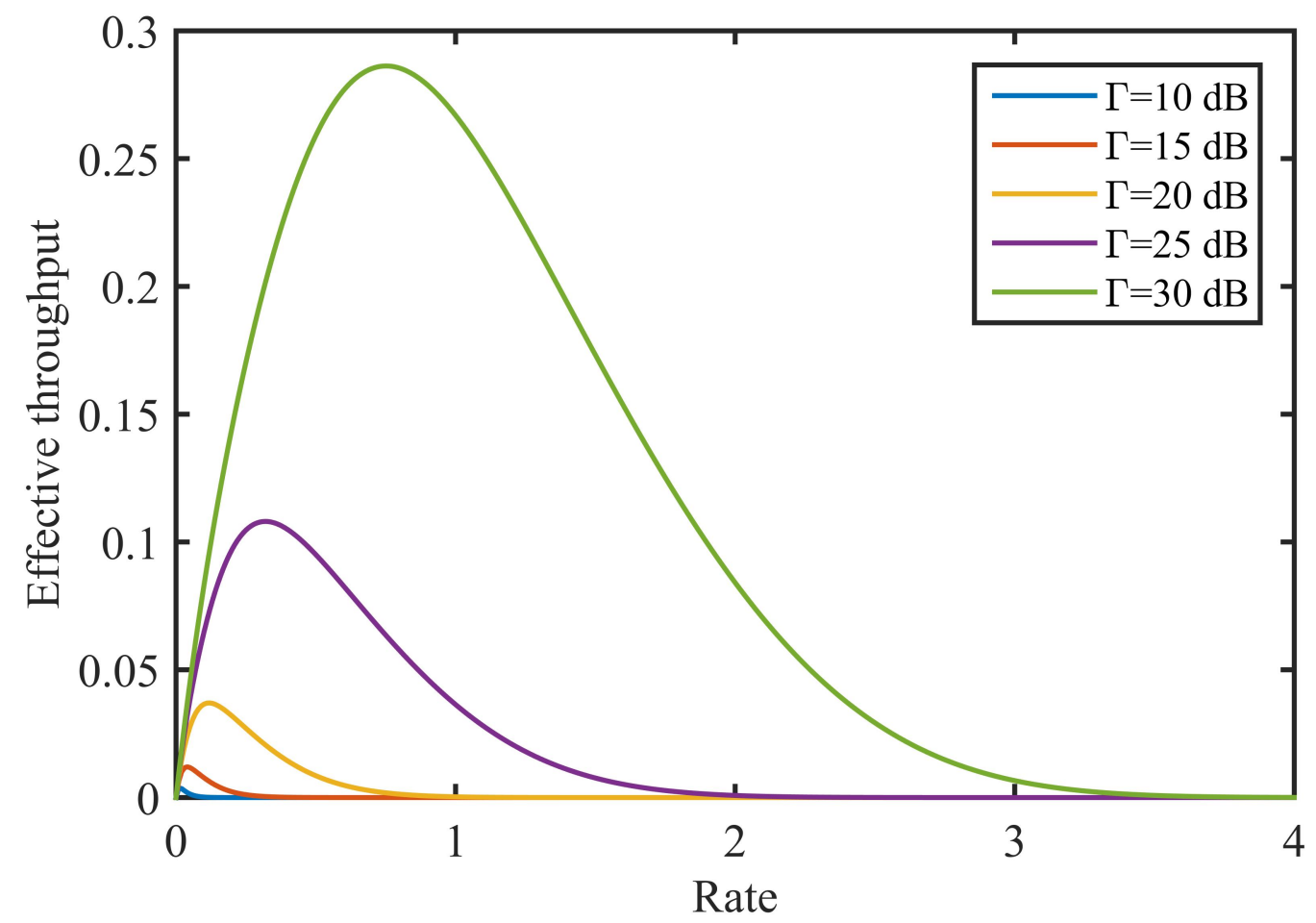

Figure 5.7: Spatially averaged effective throughput for $\alpha=3.5$ and $M=10$. 


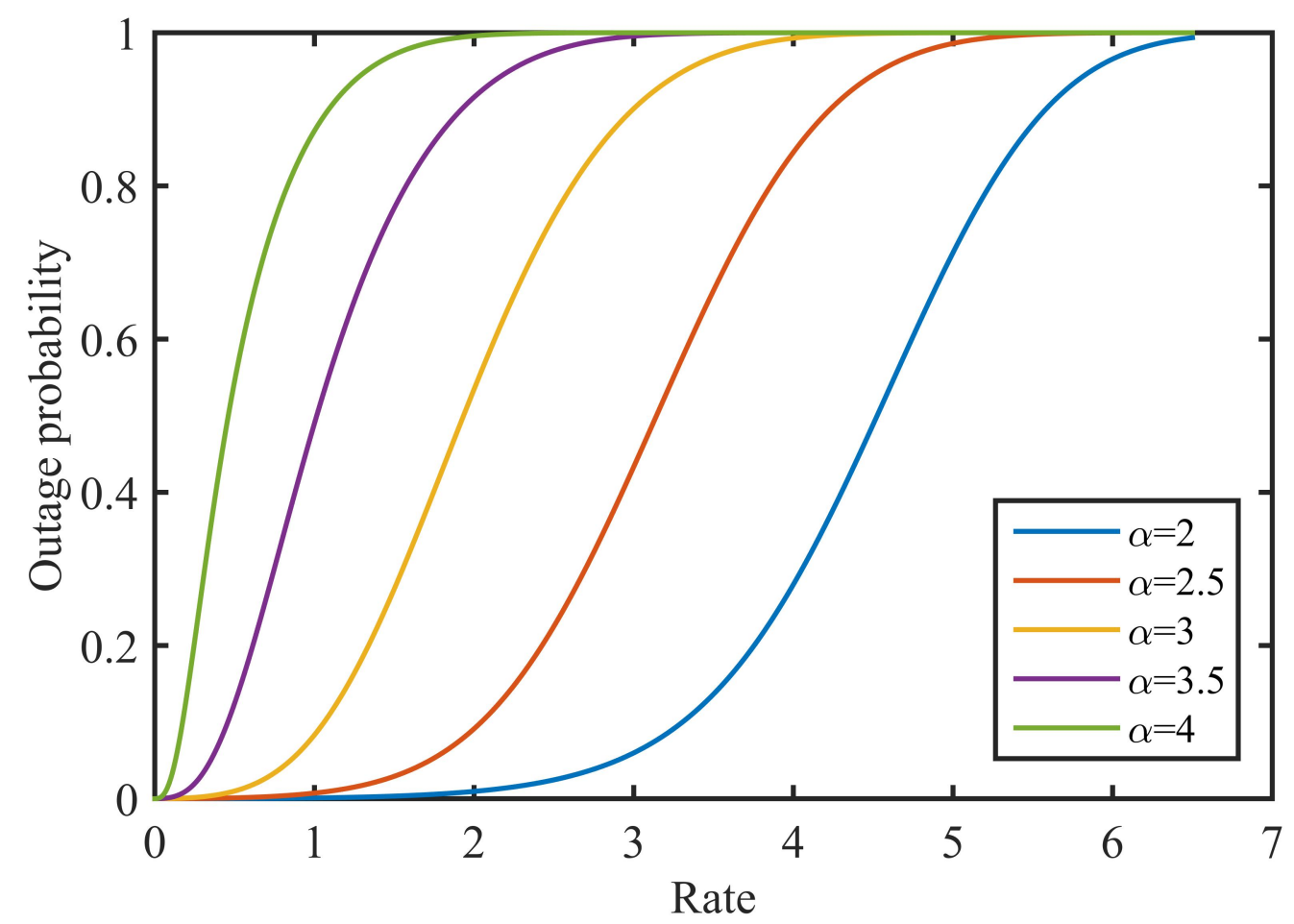

Figure 5.8: Spatially averaged outage probability for $M=10$ and $\Gamma=30 \mathrm{db}$.

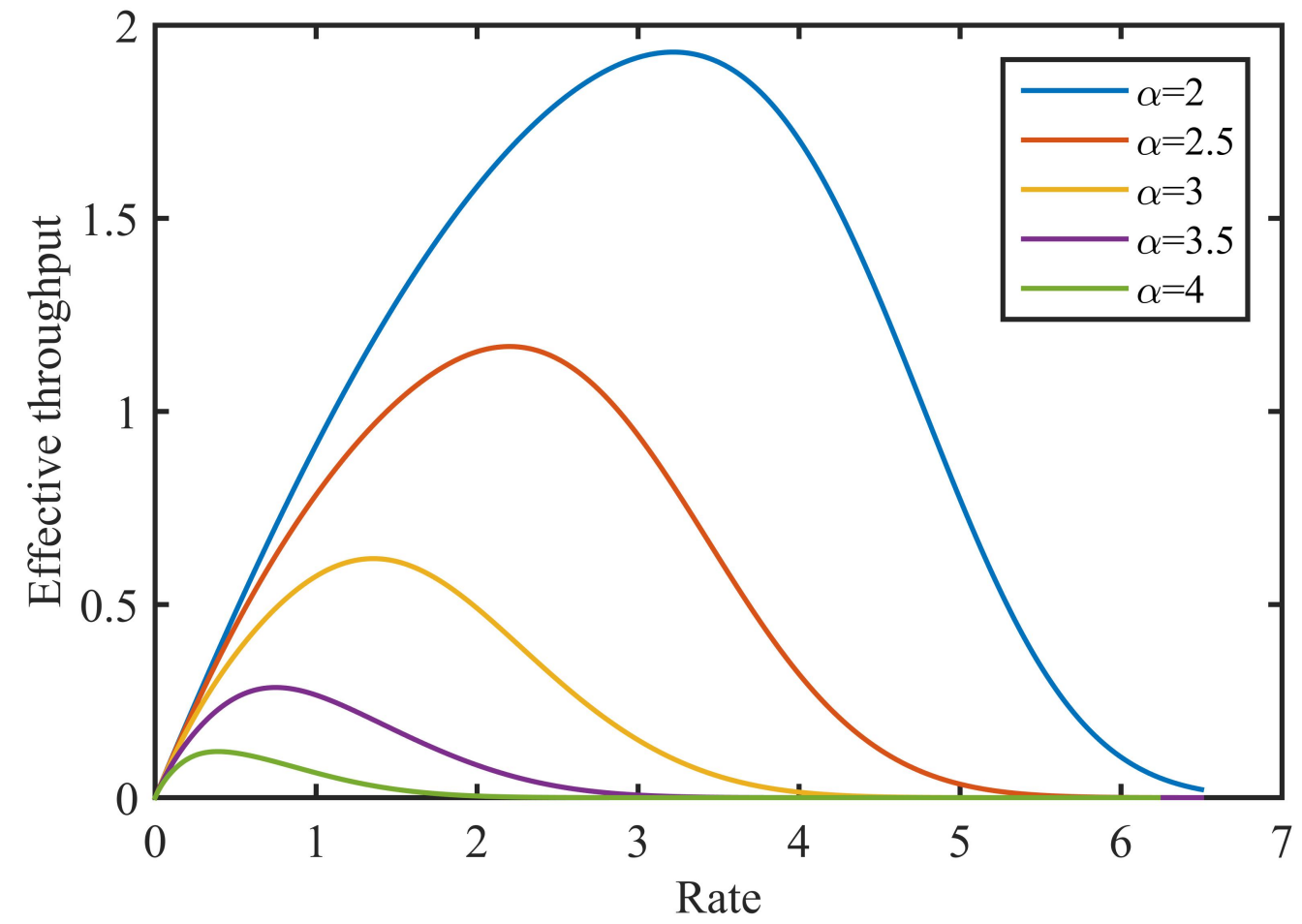

Figure 5.9: Spatially averaged effective throughput for $M=10$ and $\Gamma=30 \mathrm{db}$. 


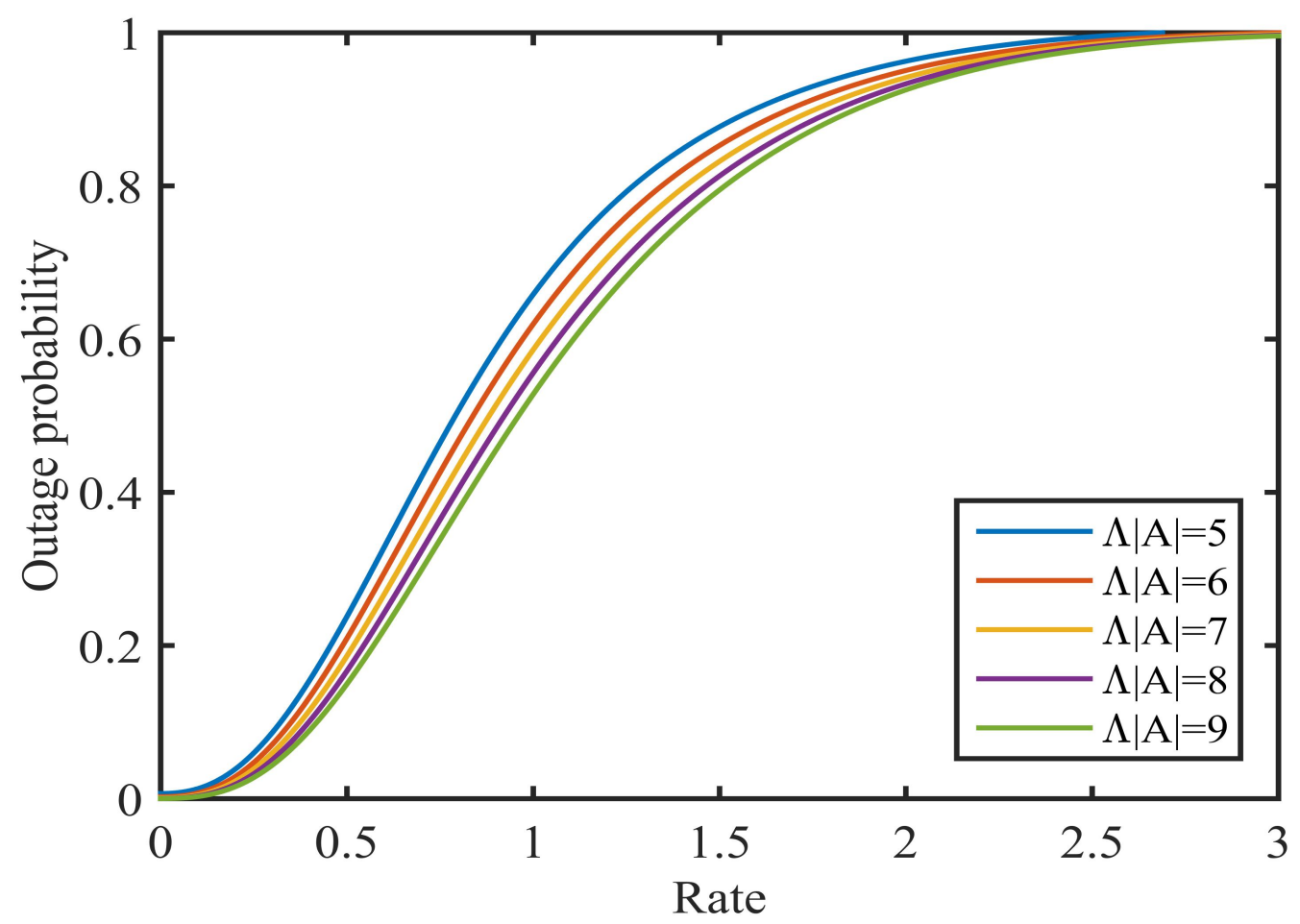

Figure 5.10: Spatially averaged outage probability for PPP.

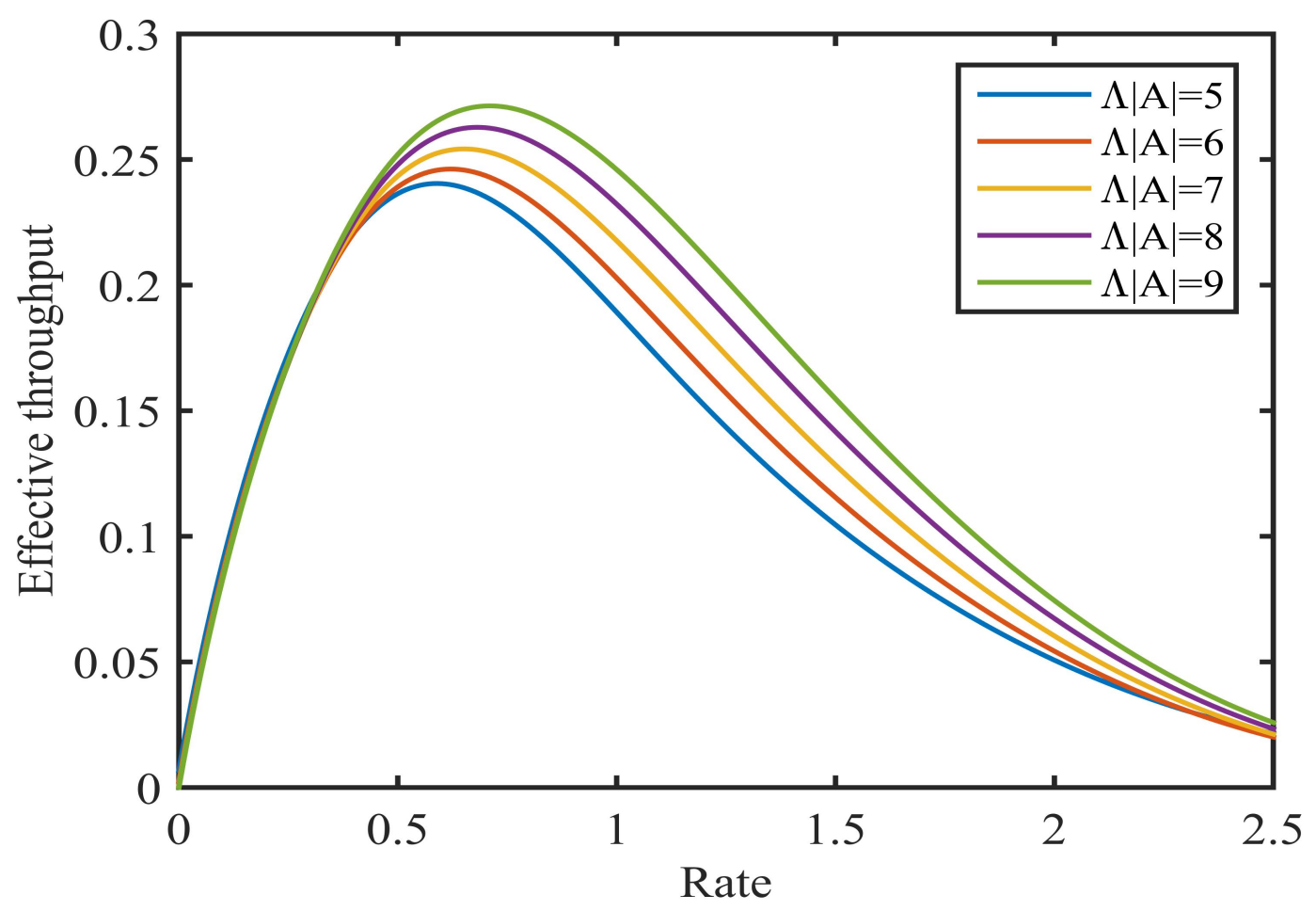

Figure 5.11: Spatially averaged $\eta_{\text {eff }}$ for PPP. 


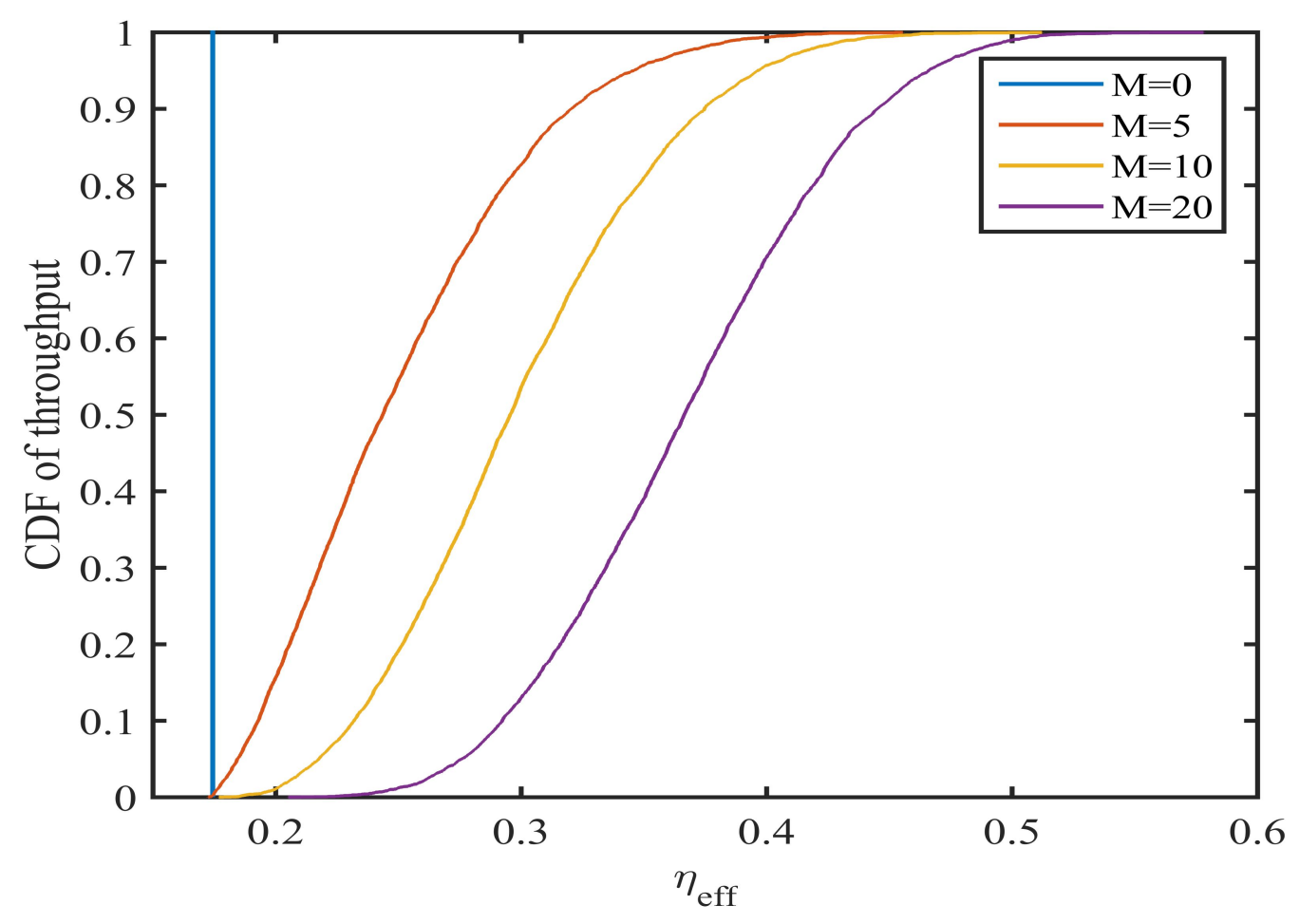

Figure 5.12: Cumulative distribution of maximum throughputs for BPP case.

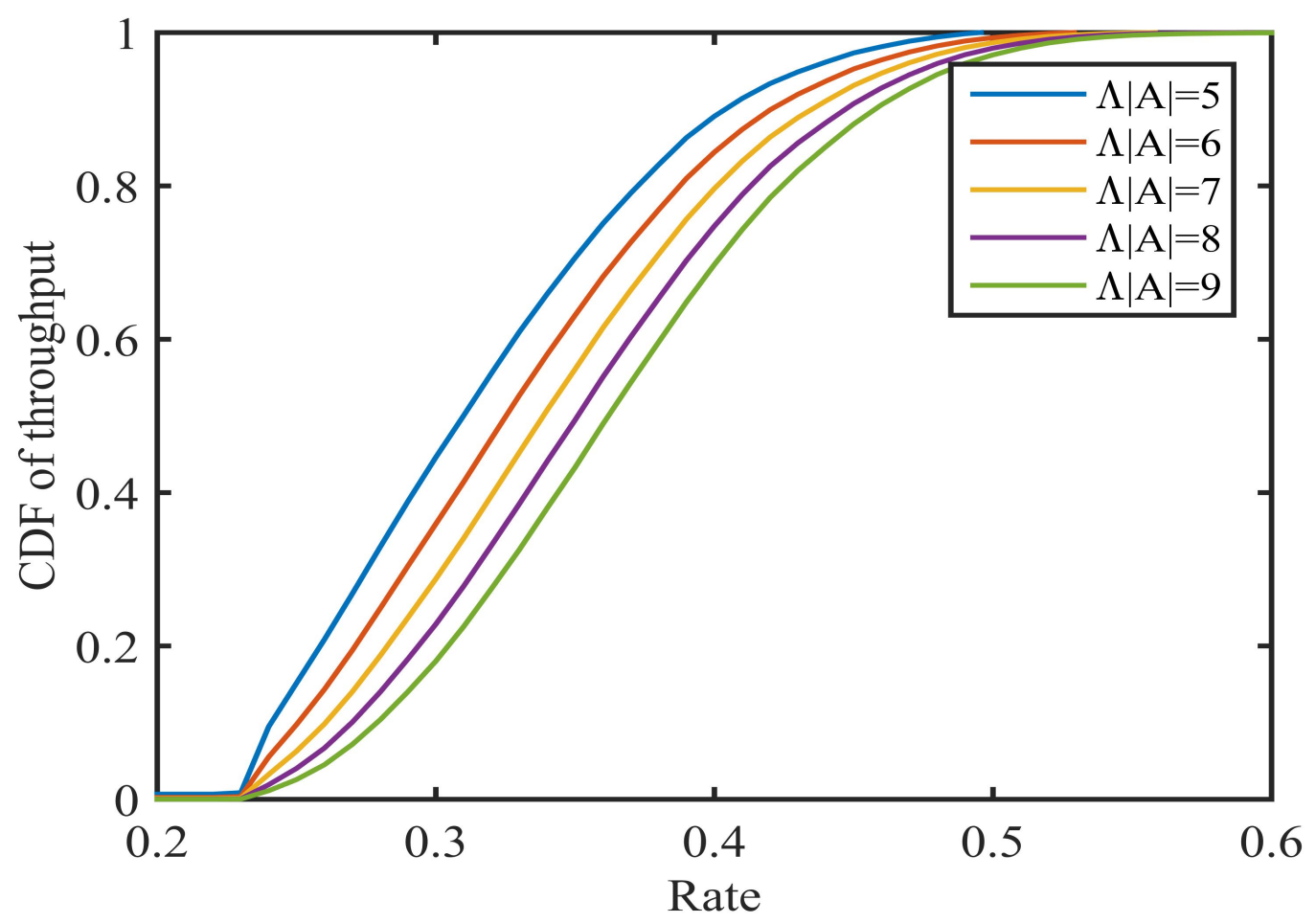

Figure 5.13: Cumulative distribution of maximum throughputs for PPP case. 


\section{Chapter 6}

\section{Conclusions and Future Work}

This chapter enlists the results that have been obtained in this thesis. The later section explains how this work can be extended and the possibility of new directions that can be explored and there is always scope for improvement, no matter how much you have worked.

\subsection{Summary of Contributions}

The major contributions of the thesis are enumerated below

1. The system model proposed is implemented using the HARQ protocol. Simulations of the system match with the analysis.

2. A Markov chain model is conceived to analyze the network and determine throughput, effective throughput and outage probability.

3. The influence of path loss exponent on spatially averaged performance metrics is determined and the results are discussed

4. A critical case when the signal to noise ratio is very low is taken up and the effect of the number of relays on the performance metrics is discussed

5. The primary performance metrics obtained are spatially averaged. First, BPP outage and BPP throughput efficiency are computed and obtained BPP metrics are marginalized with respect to number of relays to obtain PPP outage and PPP throughput efficiency. 
6. The cumulative distribution function of maximum throughput for all the network realizations is plotted as a BPP and PPP.

\subsection{Conclusions}

Based on the results obtained, significant conclusions can be drawn regarding the performance metrics and their sensitivity to the parameter variations.

- The outage performance and effective throughput improves as the number of relays increases. It has been demonstrated through results that relays help in signal transmission, reduce outage and improve throughput. In fact, a major change is observed in outage probability and effective throughput from a direct link to a relayed link.

- Increasing the $\operatorname{SNR}(\Gamma)$ improves the likelihood that the signal is successfully transmitted to the destination.

- The path loss exponent impacts the system performance. While normal range of path loss exponent is $2 \leq \alpha \leq 4$. The default value of path loss exponent considered in this thesis is 3.5. Relays are more beneficial as $\alpha$ increases because it allows for several short hops with reasonable loss rather than one hop with very high loss.

\subsection{Future Work}

While this thesis is only specific to Type-I HARQ, the same system can also be analyzed for Type-II HARQ with code combining or incremental redundancy. In Type-II HARQ with incremental redundancy, the transmitter sends extra parity bits to correct the packet instead of re-transmitting the exact same code word. Extra parity information is provided if the packet is still not decoded. Blockage probability can be incorporated into the existing model. The probability that a link is completely blocked depends on the distance and can be incorporated into the analysis. Blockage is particularly important for the mmWave systems.

The destination node features can be modified such that it can combine the information from multiple nodes and decode the original message as it does in HARBINGER. This modification results in successful transmissions with in lesser number of hops thus contributing to throughput. This thesis assumes a Rayleigh fading channel, but, the same analysis could be extended to the much complex Nakagami fading channels. 


\section{Bibliography}

[1] J. N. Laneman, D. N. C. Tse, and G. W. Wornell, "Cooperative diversity in wireless networks: Efficient protocols and outage behavior," IEEE Transactions on Information Theory, vol. 50, no. 3, pp. 3062-3080, Dec 2004.

[2] J. N. Laneman and G. W. Wornell, "Distributed space-time-coded protocols for exploiting cooperative diversity in wireless networks," IEEE Transactions on Information Theory, vol. 49, no. 10, pp. 2415-2425, Oct 2003.

[3] T. E. Hunter and A. Nosratinia, "Diversity through coded cooperation," IEEE Transactions on Wireless Communications, vol. 5, no. 2, pp. 283-289, Feb 2006.

[4] T. E. Hunter, S. Sanayei, and A. Nosratinia, "Outage analysis of coded cooperation," IEEE Transactions on Information Theory, vol. 52, no. 2, pp. 375-391, Feb 2006.

[5] M. Dianati, Xinhua Ling, K. Naik, and Xuemin Shen, "A node-cooperative ARQ scheme for wireless ad hoc networks," IEEE Transactions on Vehicular Technology, vol. 55, no. 3, pp. 1032-1044, May 2006.

[6] G. Yu, Z. Zhang, and P. Qiu, "Cooperative ARQ in wireless networks: Protocols description and performance analysis," in 2006 IEEE International Conference on Communications, June 2006, vol. 8, pp. 3608-3614.

[7] J. J. Alcaraz and J. Garcia-Haro, "Performance of single-relay cooperative ARQ retransmission strategies," IEEE Communications Letters, vol. 13, no. 2, pp. 121-123, February 2009.

[8] Bin Zhao and M. C. Valenti, "Practical relay networks: A generalization of hybridARQ," IEEE Journal on Selected Areas in Communications, vol. 23, no. 1, pp. 7-18, Jan 2005.

[9] M. Zorzi and R. R. Rao, "Geographic random forwarding (GeRaF) for ad hoc and sensor networks: multihop performance," IEEE Transactions on Mobile Computing, vol. 2, no. 4, pp. 337-348, Oct 2003.

[10] M. C. Valenti, D. Torrieri, and S. Talarico, "A direct approach to computing spatially averaged outage probability," IEEE Communications Letters, vol. 18, no. 7, pp. 11031106, July 2014. 
[11] M. C. Valenti and N. Correal, "Exploiting macrodiversity in dense multihop networks and relay channels," in 2003 IEEE Wireless Communications and Networking, 2003. WCNC 2003., March 2003, vol. 3, pp. 1877-1882 vol.3.

[12] T. Cover and El. Gamal, "Capacity theorems for the relay channel," IEEE Transactions on Information Theory, vol. 25, no. 2, pp. 572-584, Sep 1979.

[13] J Nicholas Laneman, Cooperative Diversity in Wireless Networks: Algorithms and Architectures, Ph.D. thesis, Massachusetts Institute of Technology, 2002.

[14] A. Bletsas, H. Shin, and M. Z. Win, "Cooperative communications with outage-optimal opportunistic relaying," IEEE Transactions on Wireless Communications, vol. 6, no. 9, pp. 3450-3460, September 2007.

[15] J. Hu and N. C. Beaulieu, "Closed-form expressions for the outage and error probabilities of decode-and-forward relaying in dissimilar Rayleigh fading channels," in 2007 IEEE International Conference on Communications, June 2007, pp. 5553-5557.

[16] S. S. Ikki and M. H. Ahmed, "Performance analysis of adaptive decode-and-forward cooperative diversity networks with best-relay selection," IEEE Transactions on Communications, vol. 58, no. 1, pp. 68-72, January 2010.

[17] J. G. Andrews, R. K. Ganti, M. Haenggi, N. Jindal, and S. Weber, "A primer on spatial modeling and analysis in wireless networks," IEEE Communications Magazine, vol. 48, no. 11, pp. 156-163, November 2010.

[18] Martin Haenggi, Stochastic geometry for wireless networks, Cambridge University Press, 2012.

[19] H. ElSawy, E. Hossain, and M. Haenggi, "Stochastic geometry for modeling, analysis, and design of multi-tier and cognitive cellular wireless networks: A survey," IEEE Communications Surveys Tutorials, vol. 15, no. 3, pp. 996-1019, Third 2013.

[20] Shu Lin, D. J. Costello, and M. J. Miller, "Automatic-repeat-request error-control schemes," IEEE Communications Magazine, vol. 22, no. 12, pp. 5-17, December 1984.

[21] John M Wozencraft and Michael Horstein, Coding for two-way channels, Massachusetts Institute of Technology, Research Laboratory of Electronics, 1961.

[22] JM Wozencraft and M Horstein, "Digitalised communication over two-way channels," in Fourth London Symp. Inform. Theory, London, UK, 1960.

[23] Shu Lin and P. Yu, "A hybrid ARQ scheme with parity retransmission for error control of satellite channels," IEEE Transactions on Communications, vol. 30, no. 7, pp. 17011719, July 1982.

[24] Yu-Ming Wang and Shu Lin, "A modified selective-repeat type-II hybrid ARQ system and its performance analysis," IEEE Transactions on Communications, vol. 31, no. 5, pp. 593-608, May 1983. 
[25] H. Chen, Y. Cai, W. Yang, and Y. Hu, "A novel Markov-chain-based method for throughput analysis in truncated cooperative HARQ systems," in 2013 International Conference on Wireless Communications and Signal Processing, Oct 2013, pp. 1-5.

[26] Y. H. Nam, K. Azarian, H. El Gamal, and P. Schniter, "Cooperation through ARQ," in IEEE 6th Workshop on Signal Processing Advances in Wireless Communications, 2005., June 2005, pp. 1023-1027.

[27] Bin Zhao and Matthew C Valenti, "Position-based relaying with hybrid-ARQ for efficient ad hoc networking," EURASIP Journal on wireless communications and networking, vol. 2005, no. 5, pp. 734983, 2005.

[28] K. Azarian, H. El Gamal, and P. Schniter, "On the optimality of the ARQ-DDF protocol," IEEE Transactions on Information Theory, vol. 54, no. 4, pp. 1718-1724, April 2008.

[29] H. A. Ngo and L. Hanzo, "Hybrid automatic-repeat-request systems for cooperative wireless communications," IEEE Communications Surveys Tutorials, vol. 16, no. 1, pp. 25-45, First 2014.

[30] B. Sklar, "Rayleigh fading channels in mobile digital communication systems. I. characterization," IEEE Communications Magazine, vol. 35, no. 9, pp. 136-146, Sep 1997.

[31] T. Ghanim and M. C. Valenti, "The throughput of hybrid-ARQ in block fading under modulation constraints," in 2006 40th Annual Conference on Information Sciences and Systems, March 2006, pp. 253-258. 